\title{
The Share of the Mean Turbulent Kinetic Energy in the Near-Neutral Surface Layer for High and Low Wind Speeds
}

\author{
M. Schiavon ${ }^{1,2}$ - F. Tampieri ${ }^{2} \cdot$ F. C. Bosveld ${ }^{3} \cdot$ M. Mazzola $^{2} \cdot$ S. Trini Castelli ${ }^{4}$. \\ A. P. Viola ${ }^{5}$. C. Yagüe ${ }^{6}$
}

Received: 17 April 2018 / Accepted: 5 February 2019 / Published online: 21 February 2019

(c) Springer Nature B.V. 2019

\begin{abstract}
We examine the dependence on wind speed of the share of the mean turbulent kinetic energy among the three velocity components in the near-neutral surface layer. To contrast the general behaviour and the local effects, four datasets are considered, corresponding to different surfaces and environmental conditions. For high wind speeds (i.e., wind speed $\approx 10 \mathrm{~ms}^{-1}$ ), the shares are well-defined and about the same for all sites. As wind speed decreases (becoming $\approx 1 \mathrm{~ms}^{-1}$ ), large record-to-record variability occurs giving, on average, an almost isotropic state for the horizontal velocity components. Through spectral analysis, we relate this behaviour to the low-frequency, submeso motions and to the lack of conditions required by Reynolds averaging. The implications for modelling are also discussed, showing that the wind speed, or a related quantity, must be accounted for, besides stability, in second-order closures.
\end{abstract}

Keywords Dissipation rate $\cdot$ Low wind speed $\cdot$ Submeso motions · Turbulent kinetic energy $\cdot$ Velocity spectra

\section{Introduction}

Similarity theories are of wide use in the atmospheric boundary layer (ABL). For any theory not directly based on first principles, such theories are well-behaved if their applicability con-

Electronic supplementary material The online version of this article (https://doi.org/10.1007/s10546-01900435-6) contains supplementary material, which is available to authorized users.

M. Schiavon

mario.schiavon2@unibo.it

1 Department of Physics and Astronomy, Univeristy of Bologna, Bologna, Italy

2 Institute of Atmospheric Sciences and Climate - ISAC-CNR, Bologna, Italy

3 Royal Netherlands Meteorological Institute, De Bilt, The Netherlands

4 Institute of Atmospheric Sciences and Climate - ISAC-CNR, Turin, Italy

5 Institute of Atmospheric Sciences and Climate - ISAC-CNR, Rome, Italy

6 Dpt. Física de la Tierra y Astrofísica, Facultad de CC. Físicas, Universidad Complutense de Madrid, Madrid, Spain 
ditions are satisfied. The Monin-Obukhov similarity theory (MOST) (Monin and Obukhov 1954; Monin and Yaglom 1971; Obukhov 1971) has its optimum application when unsteadiness and horizontal heterogeneity are negligible, turbulence is dominated by small-scale locally-generated eddies, and vertical turbulent fluxes are large enough to be used as scales for other quantities. However, the applicability of MOST is questionable when fluxes are small, unsteadiness and heterogeneity are no longer negligible, and non-local mechanisms affect the atmospheric flow (Troen and Mahrt 1986; Cheng and Brutsaert 2005; Ha et al. 2007; Grachev et al. 2013; Liang et al. 2014).

For instance, MOST does not apply to the statistics of the horizontal velocity components in the unstable surface layer due to the effect of large-scale, convective eddies (Kaimal 1978; Wilson 2008). But critical conditions for MOST applicability occur also with neutral or stable stratification, low wind speed, weak and intermittent small-scale turbulence (Sun et al. 2012), and significant effect of submeso motions (Vickers and Mahrt 2006; Liang et al. 2014) that cause, for example, the meandering of the velocity vector (Anfossi et al. 2005; Mahrt 2007; Mortarini et al. 2016).

According to Mahrt (2014), we consider "submeso motions" as "motions between the main turbulent eddies and smallest mesoscale motions, traditionally specified to be $2 \mathrm{~km}$ horizontal scale". Being related to many physical phenomena (Mahrt 2007), submeso motions characterize a wide range of atmospheric flows (Anfossi et al. 2005; Vickers and Mahrt 2007; Mortarini et al. 2016). Furthermore, due to their non-local origin, submeso motions do not follow surface-layer similarity, although, with typical averaging times, they can contribute significantly to observed statistics, especially when small-scale turbulence is weak. Indeed, according to some authors (e.g., Vickers and Mahrt 2003), similarity relationships should be evaluated only after filtering out the contribution from these motions. This assumes that small-scale turbulence and submeso motions are separated by a spectral gap (Vickers and Mahrt 2006, 2007; Liang et al. 2014) whose existence, however, is not a universal feature. Thus, especially in statistics containing the horizontal velocity fluctuations, this separation can be less effective and somewhat arbitrary.

Wind speed is an important parameter in determining the behaviour of turbulence (Sun et al. 2012, 2016; Van de Wiel et al. 2012; Mahrt et al. 2013, 2015; Acevedo et al. 2016). In particular, low-wind-speed conditions ("low-wind conditions", hereafter) can be identified in contrast to high-wind-speed conditions ("high-wind conditions", hereafter). Only in the latter case is classical surface-layer similarity recovered. From the fluid-dynamic point of view, flow characteristics for low-wind conditions partly resemble those of shear-free flows (see e.g., Hunt 1984). Accordingly, such conditions can be defined as the mean wind speed being lower than the characteristic (horizontal) speed of large (submeso) eddies (Zilitinkevich et al. 2006), providing evidence that "low-wind conditions" are a complex property of the flow and are not only characterized by low values of the wind speed.

The difference in turbulence features between high- and low-wind conditions has impacts on applications. Concerning weather and climate prediction, many numerical models employ parametrizations of the eddy diffusion coefficient based on the mean turbulent kinetic energy (TKE), assuming universal relationships among the TKE, the velocity variances, and the vertical momentum flux, possibly affected by stability. As far as these relationships change from high- to low-wind conditions, current parametrizations must be tuned accounting for this dependence on wind speed or, alternatively, different velocity scales should be considered.

Dispersion models need expressions for the variances of the three velocity components to model tracer trajectories according to the so-called Lagrangian approach-see Thomson (1987), to avoid the citation of the numerous extensions - or to estimate the diffusion coefficient tensor in the so-called Eulerian approach, which has its basis in Taylor (1921) (see also, 
Isichenko 1992) and has been used in many applications (e.g., Hanna 1982). Thus, beside the TKE, its share among the three velocity components is a crucial information to obtain reliable applications. Expressions for the TKE shares can be obtained from the budget equations for the velocity variances applying simplifying assumptions (such as steadiness and horizontal homogeneity) and using parametrizations for the redistribution and the viscous dissipation terms (e.g., Zilitinkevich et al. 2013). However, these parametrizations are incomplete in low-wind conditions due to the effect of submeso motions.

Based on empirical observations, we investigate turbulence features in the near-neutral surface layer and how such features vary with wind speed. To evaluate the existence of universal behaviours vs local effects we consider four datasets (Sect. 2) corresponding to different environmental conditions and surface characteristics. Specifically, we focus on:

- the identification of the stability range called "near-neutral" for our specific problem (Sects. 3.1 and 3.3);

- the definition of low- and high-wind conditions (Sect. 3.2);

- the wind-speed effect on the share of TKE among the three velocity components (Sect. 3.4) with further considerations on the shear stress (Sect. 3.5);

- the relation between the observed behaviour and spectral features (Sect. 3.6);

- the modelling implications (Sect. 4).

\section{Observations}

Four datasets are considered, concerning tower observations in the ABL: over flat and uniform terrain, from the Stable Atmospheric Boundary-Layer Experiment in Spain (SABLES98) and from Cabauw in The Netherlands; in an urban area, from the Urban Turbulence Project (UTP); in an Arctic fjord over heterogeneous surface, from the Climate Change Tower (CCT) Integrated Project. These four datasets were selected to encompass a variety of surface and environmental conditions. Because the datasets were not specifically designed for this investigation, differences exist in the data collection and processing, as well as in the duration of the time series. However, a common behaviour is found among the datasets, indicating that these differences are not determinative for this study.

For all datasets, variances and covariances are calculated using block averages with fixed averaging time: $10 \mathrm{~min}$ for the Cabauw, UTP, and CCT datasets; 5 min for the SABLES98 dataset, because this was originally intended for the study of stable conditions (Cuxart et al. 2000). These averaging times may be too short for sampling the submeso contributions (Vickers and Mahrt 2007; Acevedo et al. 2014; Sect. 3.6) but they are standard for micrometeorological datasets (Mauritsen et al. 2007). Because, often, there is no spectral gap between submeso motions and small-scale turbulence, the choice of the averaging time is somewhat arbitrary. This is an important aspect that could affect the results but, for practical reasons, we used the datasets as they were originally prepared, with their own averaging times.

Double rotation of the sonic reference system is employed for three of the four datasets to align the $x$-axis with the mean velocity vector, while triple rotation is used for the UTP dataset such that the cross-wind component of the shear stress vanishes (e.g., Kaimal and Finnigan 1994, p. 239). Although this further rotation can affect the vertical and the crosswind velocity variances (compared to the double-rotation method), former investigations on the UTP dataset have shown that its effect is generally small.

For each dataset, we selected data according to the mean wind direction, excluding records with flow through the tower. 


\subsection{Cabauw}

Cabauw $\left(52^{\circ} 58^{\prime} \mathrm{N}, 4^{\circ} 55^{\prime} \mathrm{E}\right)$ is located in the south-western part of The Netherlands (Van Ulden and Wieringa 1996; Verkaik and Holtslag 2007). The site is characterized by flat terrain, with the 213-m main tower surrounded by grassland up to distances of at least $200 \mathrm{~m}$. Tree lines, roads, and scattered villages are present a few kilometres distant from the tower. The roughness length is $z_{0} \approx 0.1 \mathrm{~m}$, the exact value depending on the wind direction (Verkaik and Holtslag 2007). A 1-year set of observations, from 1 July 2007 to 30 June 2008, is considered in this study.

Mean wind and temperature are measured at $10 \mathrm{~m}, 20 \mathrm{~m}, 40 \mathrm{~m}, 80 \mathrm{~m}, 140 \mathrm{~m}$ and $200 \mathrm{~m}$ above the ground with slow-response instruments. Sonic anemometers are deployed at $3 \mathrm{~m}$, $60 \mathrm{~m}, 100 \mathrm{~m}$ and $180 \mathrm{~m}$, with sonic levels above $3 \mathrm{~m}$ excluded in this analysis, which focuses on surface-layer observations.

\subsection{Stable Atmospheric Boundary-Layer Experiment in Spain}

SABLES98 was carried out from 10 to 28 September 1998 at the Research Centre for the Lower Atmosphere (CIBA) $\left(41^{\circ} 49^{\prime} \mathrm{N}, 4^{\circ} 56^{\prime} \mathrm{W}\right)$ (Cuxart et al. 2000; Yagüe et al. 2006). Observations from ten whole days (from 13 to 22 September) and seven nights (from 10 to 13 , and from 23 to 27 September) are considered here. The campaign site is located in the centre of an $800-\mathrm{km}^{2}$ plateau (Montes Torozos) $840 \mathrm{~m}$ above the sea level and is surrounded by fairly level and grassy plains. According to San José et al. (1985) and Cuxart et al. (2000), $z_{0} \approx 0.011 \mathrm{~m}$. Mountain ranges are present about $100 \mathrm{~km}$ from CIBA.

We analyzed observations from the 100-m main tower. Mean wind speed was measured at $3 \mathrm{~m}, 10 \mathrm{~m}, 50 \mathrm{~m}$ and $100 \mathrm{~m}$ above the ground, while wind direction was measured with wind vanes at $10 \mathrm{~m}, 20 \mathrm{~m}$ and $100 \mathrm{~m}$. Profiles of mean potential temperature were obtained from thermocouples placed at fifteen levels between 0.22 and $50 \mathrm{~m}$ (see Cuxart et al. 2000). Sonic anemometers (Solent RS2) were deployed at $5.8 \mathrm{~m}, 13.5 \mathrm{~m}$ and $32 \mathrm{~m}$.

\subsection{Urban Turbulence Project}

The Urban Turbulence Project was carried out in the outskirts of Turin $\left(45^{\circ} 10^{\prime} \mathrm{N}, 7^{\circ} 38^{\prime} \mathrm{E}\right)$, in northern Italy (Mortarini et al. 2013; Trini Castelli et al. 2014). The site is characterized by a dominance of low-wind conditions, with the tower placed over flat and grassy terrain, surrounded by buildings at a distance of about $100 \mathrm{~m}$. Trini Castelli et al. (2014) found $z_{0} \approx 0.1-1 \mathrm{~m}$, depending on the wind direction. Observations were acquired from 17 January 2007 to 19 March 2008.

Three sonic anemometers (two Gill Solent 1012R2 and one Gill Solent 1012R2A) were deployed at $5 \mathrm{~m}, 9 \mathrm{~m}$ and $25 \mathrm{~m}$ above the ground. Mean quantities, variances and covariances were obtained after linear detrending and triple rotation of the sonic reference system, such that the cross-wind component of the shear stress vanishes (Falabino and Trini Castelli 2017). Data at $5 \mathrm{~m}$ and $9 \mathrm{~m}$ are mostly representative of the roughness sublayer, while observations at $25 \mathrm{~m}$ reflect inertial-sublayer characteristics. 


\subsection{Climate Change Tower Integrated Project}

The CCT Integrated Project used a 34-m high tower installed at $\mathrm{Ny}$ - $\mathrm{Alesund}\left(79^{\circ} \mathrm{N}, 12^{\circ} \mathrm{E}\right)$, Svalbard Islands, Norway (Mazzola et al. 2016), located in an Arctic fjord in western Spitsbergen. The site is characterized by heterogeneous terrain (e.g., sea-land-ice transitions) and complex topography, with bare ground present near the tower with $z_{0} \approx 10^{-4}-10^{-3} \mathrm{~m}$ in high-wind conditions. Terrain undulations are found a few hundreds of metres from the tower and mountains up to 700-m high are a few kilometres distant. The village of $\mathrm{Ny}$-Ålesund is about $1 \mathrm{~km}$ far in the north-east direction, on the coast of the fjord.

Mean wind, temperature and humidity were measured with slow-response instruments at $2 \mathrm{~m}, 4.8 \mathrm{~m}, 10.3 \mathrm{~m}$ and $33.4 \mathrm{~m}$ above the ground. Three sonic anemometers were placed at intermediate levels, i.e., at $3.7 \mathrm{~m}, 7.5 \mathrm{~m}$ and $20.5 \mathrm{~m}$ (Gill Solent R2, Gill Solent R3 and Campbell CSAT3, respectively). We analyzed data acquired since the end of 2009 until 2017.

\section{Results}

Sections 3.1, 3.2, and 3.3 present and discuss our working definitions, concerning the identification of near-neutral conditions and the specification of low- and high-wind conditions. The first important step is to single out the wind-speed effect (our topic) from the stability effect, this task being more difficult for low- than for high-wind conditions. With the second step we go beyond the mere definition of a wind-speed category, relating wind-speed thresholds to the changing dynamics of the surface layer, due to the increasing effect of the submeso motions.

After excluding the stability effect we can focus on the wind-speed effect on the share of TKE among the three velocity components (Sect. 3.4) and on the vertical transfer of momentum (Sect. 3.5). The spectral analysis of Sect. 3.6 confirms that the behaviour observed for decreasing wind speed-i.e., the increasing scatter of the data, the average tendency toward horizontal isotropy and almost two-dimensional turbulence, and the decreasing efficiency of the vertical transport of momentum - are signatures of an increasing effect of the submeso motions on surface-layer turbulence.

The analysis of the daily occurrence of near-neutral, low-wind conditions (Sect. 3.7) suggests that, generally, they are not related to a particular time of the day and thus, probably, not to particular states of the ABL.

\subsection{Choice of the Stability Parameter}

The definition of near-neutral conditions poses issues in low-wind and weak-turbulence conditions, either employing flux-based (the Obukhov length) or gradient-based (the gradient Richardson number) stability parameters. Indeed, in these conditions, both vertical gradients (of wind speed and temperature) and fluxes (of momentum and temperature) are small and poorly determined.

The local Obukhov length (e.g., Nieuwstadt 1984) reads

$$
\Lambda \equiv-\frac{\tau^{3 / 2}}{\kappa \beta\langle w \theta\rangle},
$$

where $\tau=\sqrt{\langle u w\rangle+\langle v w\rangle}$ is the kinematic horizontal shear stress, $u, v$, and $w$ are the fluctuations of the streamwise, the cross-wind, and the vertical velocity components, respectively, 
(a) Cabauw

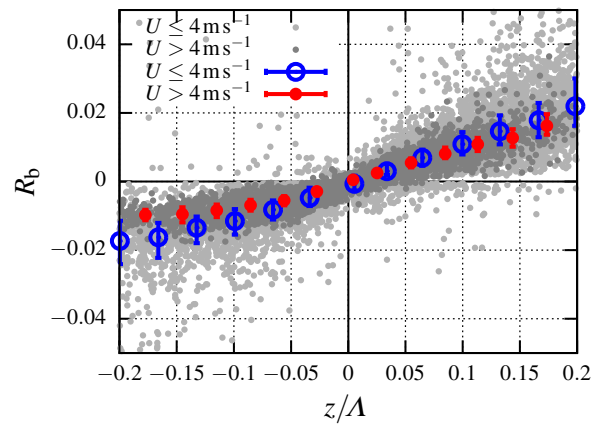

(b) SABLES98

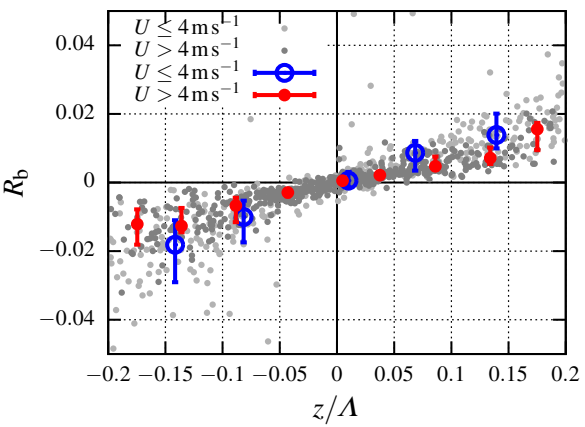

Fig. 1 Bulk Richardson number, $R_{\mathrm{b}}$ (see text), vs $z / \Lambda$ - at $3 \mathrm{~m}$ for the Cabauw dataset (a) and at $5.8 \mathrm{~m}$ for the SABLES98 dataset (b) - for wind speed at $10 \mathrm{~m}$ below and above $4 \mathrm{~ms}^{-1}$ : for the two wind-speed categories, all data points are shown (light and dark dots) along with median values (open-blue and filled-red circles) and 25th-75th percentile ranges (errorbars) in $z / \Lambda$-bins

and the brackets indicate time averaging hereafter; $\kappa=0.4$ is the von Kármán constant; $\beta \equiv g / \Theta$ is the buoyancy parameter with $g$, the acceleration due to gravity and $\Theta$, the mean potential temperature; $\langle w \theta\rangle$ is the kinematic heat flux, where $\theta$ is the temperature fluctuation around $\Theta$. All variables in Eq. 1 are measured at height $z$ and thus $\Lambda$ varies, in general, with height above the ground. The Obukhov stability parameter is $z / \Lambda$.

As an alternative stability parameter based on mean variables, we consider the bulk Richardson number (e.g., Kaimal and Finnigan 1994)

$$
R_{\mathrm{b}} \equiv \beta z \frac{\Delta \Theta\left(z_{2}, z_{1}\right)}{U^{2}(z)},
$$

where $U(z)$ is the mean wind speed (vectorial average) at the height $z$ and $\Delta \Theta\left(z_{2}, z_{1}\right)$ is the potential temperature difference between two observational levels: one at $z_{2}$ (close to $z$ ) and the other at $z_{1}$ (close to the surface). The dependence of $R_{\mathrm{b}}$ (Eq. 2) on $z_{0}$ and its asymmetry (since wind speed and temperature are not evaluated at the same levels) are not important here, because we neither compare $R_{\mathrm{b}}$ among different datasets nor use it as a discrete approximation of the gradient Richardson number. Indeed, $R_{\mathrm{b}}$ is used only to check that the indication of near-neutral conditions from $z / \Lambda$ is consistent with the temperature and wind profiles. For this, we prefer $R_{\mathrm{b}}$ over the gradient Richardson number that, as stated above, becomes ill-determined as low-wind conditions are approached (Kaimal and Finnigan 1994; Mahrt et al. 2013).

Figure 1 compares the two stability parameters for the Cabauw and SABLES98 datasets, in a stability range around neutral. For both datasets, $R_{\mathrm{b}}$ is estimated taking $z=10 \mathrm{~m}$ (and thus $U$ at $10 \mathrm{~m}$ ), while the heights for the temperature difference are $10 \mathrm{~m}$ and $2 \mathrm{~m}$ for Cabauw, and $10.88 \mathrm{~m}$ and $0.88 \mathrm{~m}$ for SABLES98. In Fig. $1, z / \Lambda$ at $3 \mathrm{~m}$ and at $5.8 \mathrm{~m}$ is considered for the Cabauw and SABLES98 datasets, respectively. Data are separated according to $U(z)$, using a wind speed of $4 \mathrm{~ms}^{-1}$ as the threshold. For each wind-speed class, all data points (light and dark grey dots) along with median values (open and filled circles) and 25th-75th percentile ranges (error bars) in $z / \Lambda$-bins are shown.

Since $R_{\mathrm{b}}$ depends on the surface characteristics (through $z_{0}$ ) and on the dataset (because of $z_{1}$ and $z_{2}$ ), the $z / \Lambda-R_{\mathrm{b}}$ relation must be considered as relative to the specific dataset instead as an absolute one. From MOST, the same $z / \Lambda-R_{\mathrm{b}}$ relationship is expected for the two wind-speed classes for a given dataset. This occurs indeed, in Fig. 1, around $z / \Lambda=0$, 
from weakly unstable to weakly stable conditions, demonstrating that $R_{\mathrm{b}}$ and $z / \Lambda$ give a consistent stability indication in this range. Supported by this result, having as primary scope the identification of near-neutral conditions, we use $z / \Lambda$ as a stability parameter, because turbulent fluxes are available for all datasets under investigation.

\subsection{Definition of Low-Wind Conditions}

We take the wind speed measured at (or near) $10 \mathrm{~m}$ (i.e., at $9 \mathrm{~m}$ for the UTP dataset) as the reference wind speed, hereinafter $U_{\mathrm{r}}$. Specifically, we use $U_{\mathrm{r}}$ to define low- and high-wind conditions and we relate its variation to the changing behaviour of the turbulence statistics. An alternative parameter is the local wind speed, $U$ (e.g., Anfossi et al. 2005; Sun et al. 2012; Mahrt et al. 2015; Mortarini et al. 2016), although Sun et al. (2012) have shown that the $U$ threshold separating the low- and the high-wind regimes depends on $z$. From the qualitative point of view, using $U$ instead of $U_{\mathrm{r}}$ does not affect the results. Since we could not assess the superiority of one velocity scale over the other, we preferred $U_{\mathrm{r}}$ to $U$, using one parameter for the whole surface layer. However, $U_{\mathrm{r}}$ is influenced by the surface roughness (changing among sites, and among seasons and wind directions at the same site) thus affecting (from a quantitative point of view) the generality of the results.

For a broad classification and for statistical evaluations we need to define thresholds for low- and high-wind conditions. Starting from the theoretical understanding of shear-free boundary-layer studies - developed in the wind tunnel and applied to the atmosphere (e.g., Zilitinkevich et al. 2006)—we identify low-wind conditions considering the ratio $\sigma_{\mathrm{H}} / U$, where $\sigma_{\mathrm{H}} \equiv \sqrt{\left\langle u^{2}\right\rangle+\left\langle v^{2}\right\rangle}$ is the horizontal velocity standard deviation and $U$ is the local mean wind speed. In this way, "low-wind" is more precisely characterized than by wind speed alone. Since submeso motions contribute significantly to $\sigma_{\mathrm{H}}$ (Vickers and Mahrt 2007; Acevedo et al. 2014; Sect. 3.6), $\sigma_{\mathrm{H}} / U$ is related to the relative strength of the submeso motions compared to that of the mean flow. In particular, low-wind conditions are defined to occur when $\sigma_{\mathrm{H}} / U$ is at least twice its value at high wind speed, $\left[\sigma_{\mathrm{H}} / U\right]_{\infty}$, i.e., when

$$
P \equiv \frac{\sigma_{\mathrm{H}} / U}{\left[\sigma_{\mathrm{H}} / U\right]_{\infty}} \geq 2
$$

For each dataset and measurement level, we obtained $\left[\sigma_{\mathrm{H}} / U\right]_{\infty}$ by plotting $\sigma_{\mathrm{H}} / U$ vs $U_{\mathrm{r}}$ and taking the value corresponding to the highest $U_{\mathrm{r}}$ available (Online Resource 1). The normalization by $\left[\sigma_{\mathrm{H}} / U\right]_{\infty}$ accounts for the dependence of $\sigma_{\mathrm{H}} / U$ on the site (through surface roughness) and on the measurement level (through $z$ ). The stability dependence of $\sigma_{\mathrm{H}} / U$ was also observed, although with differences among the datasets (see Online Resource 1). Because the estimation of $\left[\sigma_{\mathrm{H}} / U\right]_{\infty}$ is difficult for non-neutral stratification due to the small number of corresponding high-wind-speed data, we evaluated $P$ only for near-neutral conditions that, furthermore, are the focus of this study. In these conditions, if MOST is satisfied, $\sigma_{\mathrm{H}} / U=$ constant and $P=1$ independently of $U_{\mathrm{r}}$.

Plots of $P$ against $U_{\mathrm{r}}$ are presented in Fig. 2 for $|z / \Lambda|<0.03$, with data binned in $U_{\mathrm{r}}$. For each dataset, bin-medians are shown for all the measurement levels while, for the sake of clarity, data variability (i.e., 25th-75th percentile range) is shown for one level only (shaded area). As in other figures, the median value and the 25th-75th percentile range are shown only for bins containing at least 10 data points.

As was verified for each dataset by plotting $\sigma_{\mathrm{H}}$ vs $U$, the increase in $P$ for small and diminishing $U_{\mathrm{r}}$ is physical and is due to the non-vanishing $\sigma_{\mathrm{H}}$, which is a well recognized feature of ABL flows (Smith and Abbott 1961; Hanna 1983; Anfossi et al. 2005; Mahrt 2007; 
(a) Cabauw

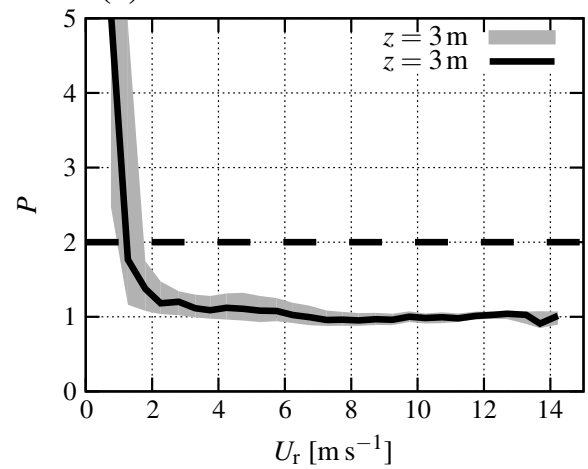

(c) UTP

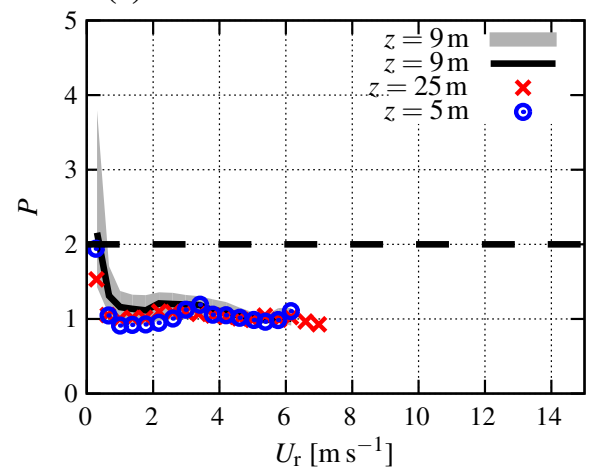

(b) SABLES98

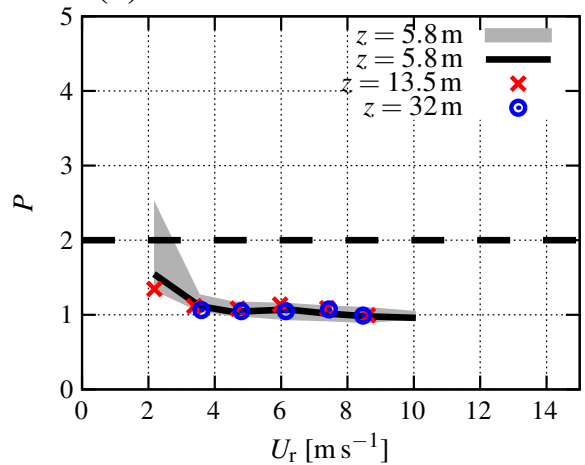

(d) $\mathrm{CCT}$

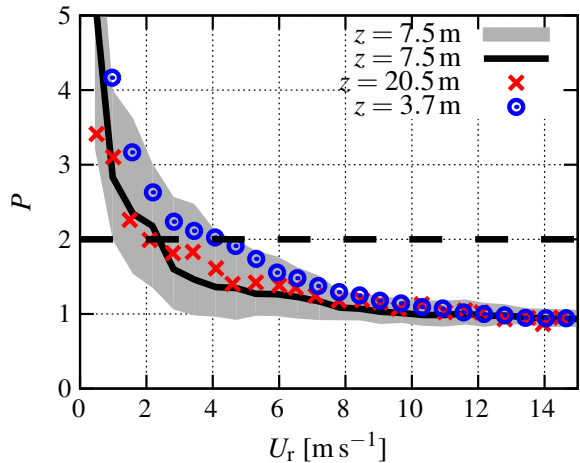

Fig. $2 P$ versus reference wind speed, $U_{\mathrm{r}}$, for near-neutral conditions: median values (points and solid line) and 25 th-75th percentile range (shaded area, for one level only). The low-wind threshold, i.e. $P=2$, is also indicated

Sun et al. 2012, 2016; Mahrt et al. 2015; Acevedo et al. 2016; Mortarini et al. 2016) attributed to the effect of submeso motions. In our case, $\sigma_{\mathrm{H}} \approx 0.1-1 \mathrm{~m} \mathrm{~s}^{-1}$ for $U \rightarrow$ zero, the exact value depending on the dataset. Thus, the magnitude of the horizontal velocity fluctuations is usually higher than the instrumental sensitivity also in low-wind conditions. However, in these conditions, Taylor's hypothesis of frozen turbulence (e.g., Stull 1988) is probably no longer satisfied because $\sigma_{\mathrm{H}} / U \gtrsim 0.5$.

The low-wind threshold (for which $P=2$ ) changes among sites, ranging from $U_{\mathrm{r}} \approx$ $0.5 \mathrm{~m} \mathrm{~s}^{-1}$ for the UTP site to $U_{\mathrm{r}} \approx 2-4 \mathrm{~m} \mathrm{~s}^{-1}$ for the CCT site, the latter showing larger scatter and a more gentle variation of $P$ with respect to the others. Following strictly our criterion, the low-wind threshold cannot be fairly established for the SABLES98 site because of the few low-wind data (Fig. 2b). However, also for this site, $P$ is significantly $>1$ (with $P>1.5$ for half of the cases) already for $U_{\mathrm{r}} \approx 3 \mathrm{~m} \mathrm{~s}^{-1}$.

According to Fig. 2, we used different thresholds for different sites. However, for each site, the same threshold was assumed for all measurement levels because no significant heightdependence was found: even for the CCT site, the difference between the $U_{\mathrm{r}}$-threshold at $z=3.7 \mathrm{~m}$ and that at the two upper levels is comparable with the spread of the data. We chose the high-wind thresholds in a wind-speed region with $P \approx 1$. Low- and high-wind thresholds for each site are reported in Table 1. For practical reasons, the thresholds are chosen also for 
Table 1 Definition of low- and high-wind conditions

\begin{tabular}{lll}
\hline Dataset & Low-wind $\left(\mathrm{m} \mathrm{s}^{-1}\right)$ & High-wind $\left(\mathrm{m} \mathrm{s}^{-1}\right)$ \\
\hline Cabauw & $U_{\mathrm{r}}<2$ & $U_{\mathrm{r}}>8$ \\
SABLES98 & $U_{\mathrm{r}}<3$ & $U_{\mathrm{r}}>8$ \\
UTP & $U_{\mathrm{r}}<0.5$ & $U_{\mathrm{r}}>3$ \\
CCT & $U_{\mathrm{r}}<2$ & $U_{\mathrm{r}}>8$ \\
\hline
\end{tabular}

retaining enough low- and high-wind data: thus the low-wind threshold for the Cabauw and SABLES98 sites is slightly higher than that suggested by Fig. 2, and the high-wind threshold for the UTP site is lower than the others. This, however, has no significant effect on the results, which do not depend on the exact determination of the thresholds, provided that $P$ is significantly $>1$ for the low-wind data (thus the value of 2 in Eq. 3 ) and $P \approx 1$ for the high-wind data.

It is not straightforward to relate the observed low-wind thresholds to the surface characteristics, both at the local scale and at the mesoscale, beside the fact that more datasets should be considered for this. Concerning the dependence on the roughness length (Mahrt et al. 2013), no clear conclusions can be drawn in our case. For instance, the small low-wind threshold observed for the UTP site is consistent with the large $z_{0}$ but could also be determined by the damping effect of the buildings on the submeso motions (Mortarini et al. 2016). Furthermore, the low-wind threshold is similar for the CCT, Cabauw, and SABLES98 sites, although $z_{0}$ for the first one is at least one order of magnitude smaller than that of the other two. A direct comparison among the UTP site and the others is in any case difficult, since in the urban environment even the role of $z$ and $z_{0}$ should be interpreted carefully, because of the combined influence of the local surface and of the distant buildings (Trini Castelli et al. 2014).

The low-wind threshold derived from the $P$-criterion is sensitive to the averaging time. In particular, it increases with extending averaging time because of the augmenting submeso contribution to $\sigma_{\mathrm{H}}$ (Anfossi et al. 2005; Vickers and Mahrt 2007; Acevedo et al. 2014; Sect. 3.6). We verified this for the CCT site (at $z=7.5 \mathrm{~m}$ ) by estimating $\sigma_{\mathrm{H}}$ for different averaging times with the multiresolution decomposition (Howell and Mahrt 1997; Howell and Sun 1999; Vickers and Mahrt 2003). To test for longer averaging times, we considered 30-min records, thus using 30-min averages for $U, U_{\mathrm{r}}$, and for the turbulent fluxes used to identify near-neutral conditions (i.e., $|z / \Lambda|<0.03$ ). The low-wind threshold increased from 2.2 to $2.7 \mathrm{~m} \mathrm{~s}^{-1}$ as the averaging time extended from $\approx 400$ to $\approx 1600 \mathrm{~s}$. This shows that the averaging time has only a limited effect on our results. For averaging times $\lesssim 200 \mathrm{~s}, P>2$ was never reached, on average, although $P$ still increased as $U_{\mathrm{r}} \rightarrow$ zero.

\subsection{Remarks on the Stability Effects on the TKE Shares}

Let us define the TKE shares among the streamwise, $u$, the cross-wind, $v$, and the vertical velocity component, $w$, as

$$
A_{\alpha} \equiv \frac{\sigma_{\alpha}^{2}}{2 E_{\mathrm{K}}}
$$

where $\alpha=u, v, w, \sigma_{\alpha}^{2} \equiv\left\langle\alpha^{2}\right\rangle$, and $E_{\mathrm{K}} \equiv\left(\sigma_{u}^{2}+\sigma_{v}^{2}+\sigma_{w}^{2}\right) / 2$ is the TKE. 
(a) Cabauw $z=3 \mathrm{~m}$

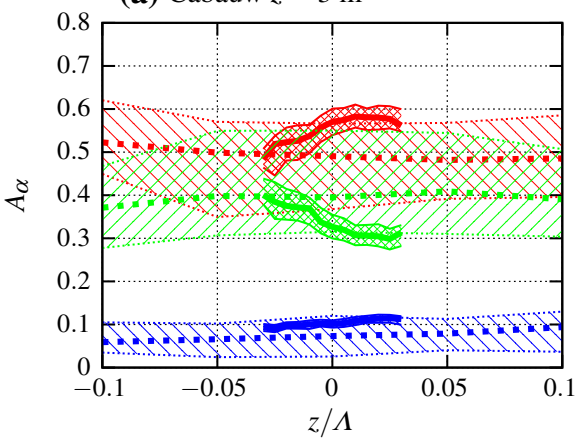

(c) $\mathrm{UTP} z=9 \mathrm{~m}$

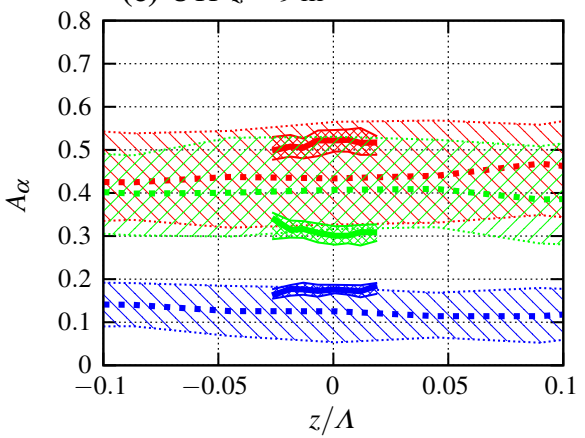

(b) SABLES98 $z=5.8 \mathrm{~m}$

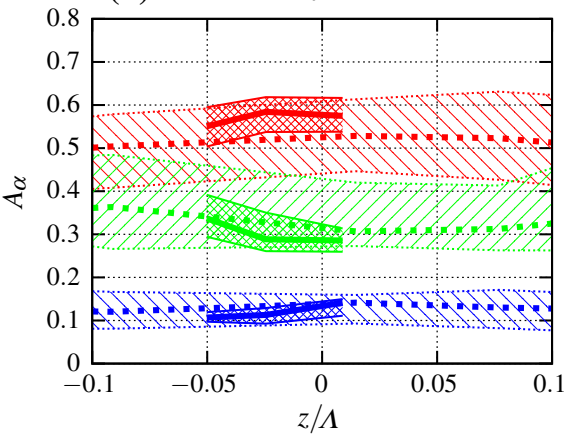

(d) $\mathrm{CCT} z=7.5 \mathrm{~m}$

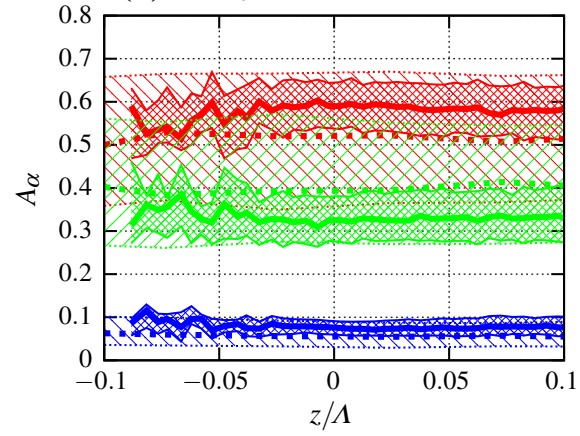

Fig. 3 TKE shares, $A_{\alpha}$, for $\alpha=u$ (red), $v$ (green), and $w$ (blue) versus $z / \Lambda$ for low- and high-wind conditions (Table 1). Median and 25th-75th percentile range are shown: solid line and heavy shading for high-wind regime; dashed line and light shading for low-wind regime. For high-wind conditions, the represented stability range changes among datasets because of the different number of available data. (For the other measurement levels see Online Resource 2)

The stability effect on the TKE shares is well assessed in the literature (Tampieri 2017). Here, we evaluate the dependence of $A_{\alpha}$ on $z / \Lambda$ to identify the near-neutral stability range, for which $A_{\alpha}(z / \Lambda) \approx A_{\alpha}(z / \Lambda=0)$. Considering one measurement level for each dataset, Fig. 3 shows $A_{\alpha}$ vs $z / \Lambda$ in a narrow range around neutral, for low- and high-wind conditions as defined in Table 1. Accounting for the larger scatter of the data, the $z / \Lambda$-bins are wider for the low-wind than for the high-wind category. Thus, the stability range spanned by the shaded areas in Fig. 3 is not representative for the relative $z / \Lambda$-distribution of low- and high-wind data: even for the Cabauw, SABLES98, and UTP datasets there are high-wind data points outside the stability range covered by the shaded area.

In the considered range of $z / \Lambda$, from weakly convective to weakly stable conditions:

- For a given $z / \Lambda$, the variability of $A_{\alpha}$ is smaller for high-wind than for low-wind conditions, for all sites and measurement levels.

- For high-wind conditions and $z / \Lambda \approx 0, A_{\alpha}$ is different for the three velocity components and similar for all sites and measurement levels. The only exception is the UTP site at $z=5 \mathrm{~m}$, for which $A_{u} \approx A_{v}$ even for high-wind conditions (see Online Resource 2).

- For high-wind conditions and $z / \Lambda<0$, a tendency towards horizontal isotropy (i.e., $\left.A_{u}=A_{v}\right)$ is detected as more unstable conditions are approached for the homogeneous 
sites of Cabauw and SABLES98. This occurs at smaller $|z / \Lambda|$ for the Cabauw site (Fig. 3a).

- For low-wind conditions, the stability dependence of $A_{\alpha}$ is weak or almost undetectable for $|z / \Lambda| \leq 0.2$. Considering also the scatter of the data, and despite the critical interpretation of stability parameters in low-wind conditions (Trini Castelli et al. 2014), this is consistent with a dominance of non-MOST effects (such as submeso motions) on surface-layer turbulence (Vickers and Mahrt 2003, 2007; Anfossi et al. 2005; Moraes et al. 2005; Sun et al. 2012; Acevedo et al. 2014; Mahrt et al. 2015; Mortarini et al. 2016).

According to the above considerations and the results presented in Fig. 3, the near-neutral stability range used in this analysis is $|z / \Lambda|<0.03$, which is consistent with both theoretical and empirical evidences (Kaimal and Finnigan 1994; Pahlow et al. 2001; Moraes et al. 2005; de Franceschi et al. 2009; Trini Castelli and Falabino 2013; Trini Castelli et al. 2014; Tampieri 2017). This interval is further restricted for the Cabauw dataset, taking $-0.01<z / \Lambda<0.03$, to exclude the observed unstable-range effect.

Although the onset of near-neutral conditions is usually related to strong winds, low-wind almost-neutral cases occur too, for instance favoured by the cloud coverage (Mahrt et al. 2015).

\subsection{The Wind-Speed Effect on the TKE Shares}

Figure 4 shows the TKE shares against $U_{\mathrm{r}}$ for near-neutral conditions. The overall behaviour is similar among the different sites and measurement levels, including the high-wind values of $A_{\alpha}, A_{\alpha \infty}$, (Table 2), with the only aforementioned exception of the UTP site at $z=$ $5 \mathrm{~m}$. Generally, $A_{v \infty} \approx 0.3$, in agreement with Zilitinkevich et al. (2013), whereas larger differences among datasets concern the streamwise and the vertical share, with $A_{u \infty} \approx 0.5-$ 0.6 and $A_{w \infty} \approx 0.1-0.2$, depending on the site. The UTP site high-wind values at $9 \mathrm{~m}$ and $25 \mathrm{~m}$ are close to those used by Zilitinkevich et al. (2013), while $A_{w \infty}$ is particularly small for the CCT site (Table 2). The triple rotation employed only for the UTP dataset can contribute to the differences with respect the other datasets. Whereas the small $A_{w \infty}$ at the CCT site is consistent with a large submeso contribution (Sect. 3.6) favoured by the heterogeneity of the surface at the mesoscale: i.e., mountains and slopes a few kilometres from the tower.

As $U_{\mathrm{r}}$ decreases, the median values of $A_{u}$ and $A_{v}$ become closer-indicating a tendency toward horizontal isotropy - and the scatter of the data increases-indicating a larger recordto-record variability for the same dataset (Fig. 4). This variability is particularly large at the CCT site, probably because of its heterogeneity. Instead, the already mentioned buildings effect (Sect. 3.2) can explain why the variability at the UTP site is comparable to that at the more homogeneous sites of SABLES98 and Cabauw.

At the Cabauw and CCT sites, for $U_{\mathrm{r}}<1 \mathrm{~m} \mathrm{~s}^{-1}$, the distribution of $A_{u}$ and $A_{v}$ becomes very skewed and $A_{u}<A_{v}$ considering median values (Fig. 4a and d). We have no explanation for this behaviour, although doubts on its significance are legitimate because the scatter of the data is large and this occurs only at $z=7.5 \mathrm{~m}$ for the CCT site (Online Resource 2).

Regarding the 25th-75th percentile range shown in Fig. 4 and its dependence on $U_{\mathrm{r}}$, the main variation concerns the lower boundary of $A_{u}$ and the upper boundary of $A_{v}$, with the ranges of $A_{u}$ and $A_{v}$ that almost completely overlap for low wind speed. This is consistent with an increasing variability of the wind direction for decreasing wind speed, which is a well recognized feature of ABL flows (Joffre and Laurila 1988; Hanna 1990; Anfossi et al. 2005; Mahrt 2007; Mahrt et al. 2012; Mortarini et al. 2016). Indeed, a larger $A_{v}$ (and thus 
(a) Cabauw $z=3 \mathrm{~m}$

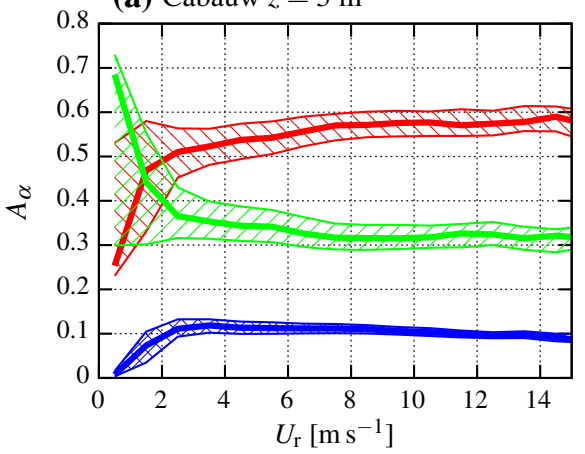

(c) UTP $z=9 \mathrm{~m}$

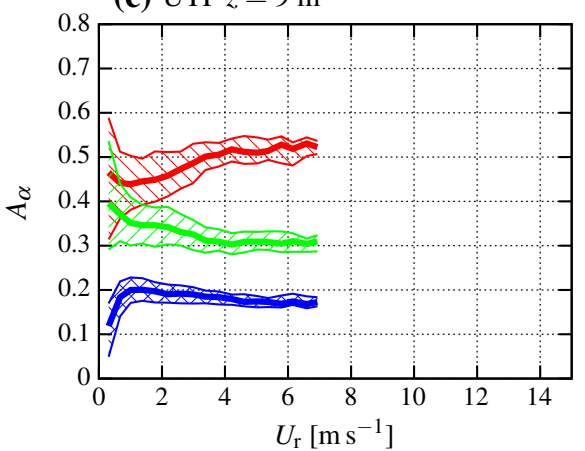

(b) SABLES $98 z=5.8 \mathrm{~m}$

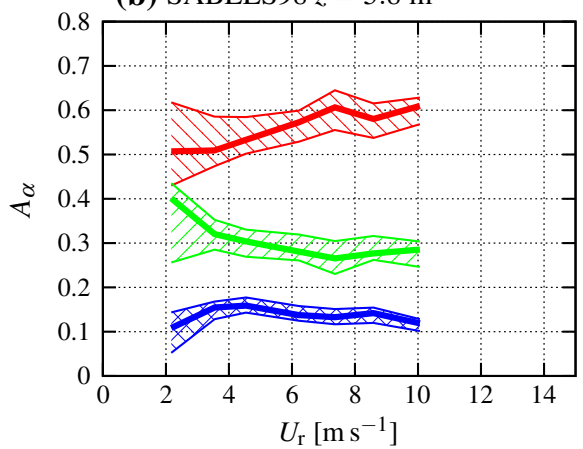

(d) CCT $z=7.5 \mathrm{~m}$

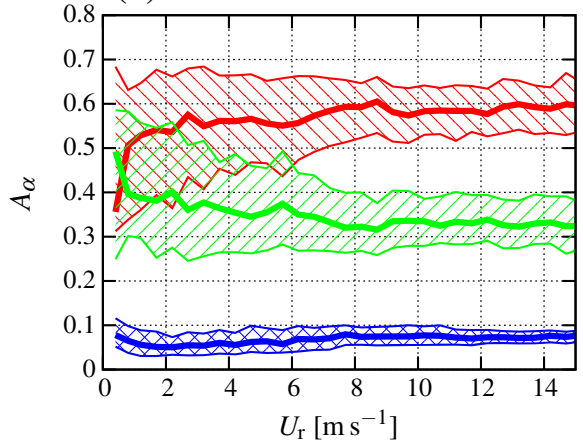

Fig. 4 TKE share, $A_{\alpha}$, versus reference wind speed, $U_{\mathrm{r}}$, for near-neutral conditions: $\alpha=u$ (red), $v$ (green), and $w$ (blue); median value and 25th-75th percentile range (for the other measurement levels see Online Resource 3)

Table 2 Average shares for high-wind conditions

\begin{tabular}{lccccc}
\hline Dataset & $z(\mathrm{~m})$ & $A_{u \infty}$ & $A_{v \infty}$ & $A_{w \infty}$ & Number of data \\
\hline Cabauw & 3 & 0.57 & 0.32 & 0.11 & 2075 \\
SABLES98 & 5.8 & 0.58 & 0.30 & 0.12 & 88 \\
& 13.5 & 0.56 & 0.30 & 0.14 & 43 \\
& 32 & 0.49 & 0.33 & 0.18 & 17 \\
UTP & 5 & 0.46 & 0.39 & 0.15 & 557 \\
& 9 & 0.52 & 0.31 & 0.17 & 673 \\
& 25 & 0.54 & 0.28 & 0.18 & 484 \\
CCT & 3.7 & 0.60 & 0.32 & 0.08 & 2436 \\
& 7.5 & 0.59 & 0.34 & 0.08 & 6652 \\
& 20.5 & 0.57 & 0.34 & 0.09 & 821 \\
Z13 & - & 0.5 & 0.3 & 0.2 & -
\end{tabular}

Values from Zilitinkevich et al. (2013) (their neutral limit, here Z13) are also reported 

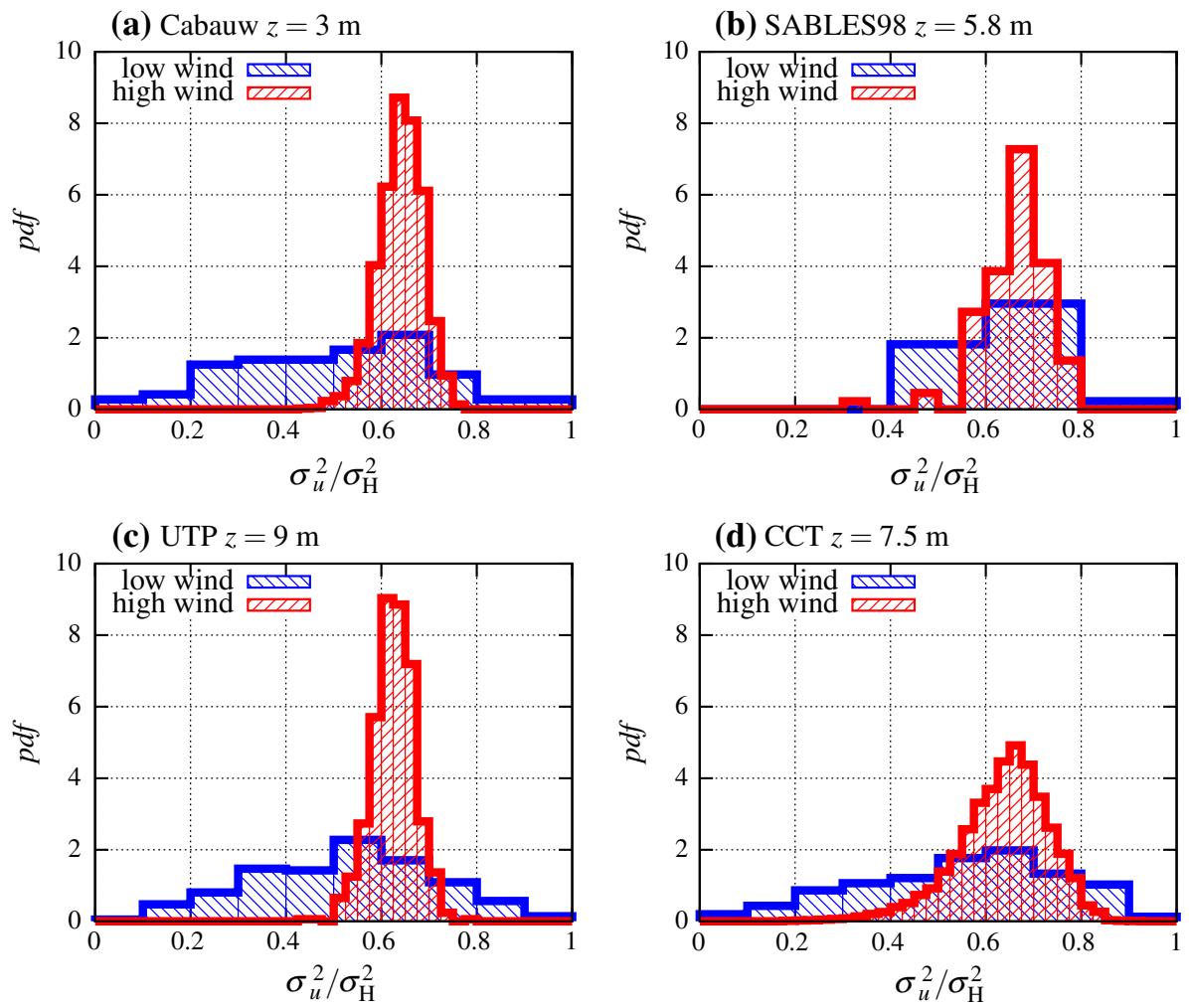

Fig. 5 Probability density functions $(p d f)$ of $\sigma_{u}^{2} / \sigma_{\mathrm{H}}^{2}$ (i.e., the relative amount of horizontal TKE in the streamwise component) for low- and high-wind conditions (for the other measurement levels see Online Resource 4)

a smaller $A_{u}$ ) corresponds to a larger variability of the wind direction during the averaging time (not shown).

At all sites except the CCT site, after a slight increase for decreasing wind speed, $A_{w}$ decreases as $U_{\mathrm{r}} \rightarrow$ zero (Fig. 4a-c). This transition towards almost two-dimensional turbulence suggests a dominant submeso contribution and is consistent with Mortarini et al. (2016) who related the decrease in $\sigma_{w} / \sigma_{\mathrm{H}}$ to the occurrence of meandering conditions. For the CCT site, $A_{w}$ is almost independent of wind speed (Fig. 4d) but is also small compared to the value at the other sites: the CCT site $A_{w \infty}$-value being lower than the SABLES98 and UTP site low-wind values. For all sites, the spread of $A_{w}$ is small, the record-to-record variability concerning mainly the horizontal shares. For the SABLES98 and UTP sites, $A_{w \infty}$ increases with $z$ (Table 2) in agreement with Högström et al. (2002) and with the decreasing damping effect of the ground on the large-scale vertical velocity fluctuations (e.g., Olesen et al. 1984).

The value of $U_{\mathrm{r}}$ corresponding to the decrease in $A_{w}$ and/or the overlap between $A_{u}$ and $A_{v}$ is close to the low-wind threshold identified in Fig. 2 being (Fig. 4 and Online Resource 3): $U_{\mathrm{r}} \approx 2 \mathrm{~m} \mathrm{~s}^{-1}$ for the Cabauw site; $U_{\mathrm{r}} \approx 1 \mathrm{~m} \mathrm{~s}^{-1}$ at all measurement levels for the UTP site; $U_{\mathrm{r}} \approx 2 \mathrm{~m} \mathrm{~s}^{-1}$ and $3 \mathrm{~m} \mathrm{~s}^{-1}$ at $5.8 \mathrm{~m}$ and $13.5 \mathrm{~m}$, respectively, for the SABLES98 site (there are not enough data at $32 \mathrm{~m}$ ); $U_{\mathrm{r}} \approx 2 \mathrm{~m} \mathrm{~s}^{-1}, 4 \mathrm{~m} \mathrm{~s}^{-1}$ and $6 \mathrm{~m} \mathrm{~s}^{-1}$ at 
$3.7 \mathrm{~m}, 7.5 \mathrm{~m}$ and $20.5 \mathrm{~m}$, respectively, for the CCT site. Furthermore, the interchange between $A_{u}$ and $A_{v}$ as $U_{\mathrm{r}}$ decreases is more gradual than the decrease in $A_{w}$ and the increase in $\sigma_{\mathrm{H}} / U$.

To investigate further the share of the horizontal velocity variance between the two horizontal components and its dependence on wind speed, we calculated the probability density function (p.d.f.) of $\sigma_{u}^{2} / \sigma_{\mathrm{H}}^{2}$ for low- and high-wind conditions, defined according to Table 1 . Figure 5 shows the p.d.f. also for the SABLES98 dataset, although statistical significance is reduced by the small number of low-wind records. For high-wind conditions, the p.d.f. is narrow for the Cabauw, SABLES98 and UTP datasets (Fig. 5a-c) and, although broader, also the CCT p.d.f. has a well-defined maximum (Fig. 5d). Instead, at all sites and measurement levels, the p.d.f. is almost flat for low-wind conditions, indicating that the share of the horizontal velocity variance between the $u$ and $v$ components is almost unpredictable (as is the direction of the mean flow) and, in each single realization, may be quite different from the median value.

By summarizing, as wind speed decreases, the range of values assumed by the horizontal shares widens and turbulence changes from a definite horizontal anisotropy (with $A_{u} \approx$ 0.6 and $A_{v} \approx 0.3$ ) to an almost isotropic condition, on average, but with large recordto-record variability. We exclude that instrumental errors are the cause of this behaviour because, as already noted, the magnitude of the horizontal velocity fluctuations is higher than the instrumental sensitivity even in low-wind conditions. Instead, in these conditions, the horizontal shares are less dependent on the wind direction because the flow is significantly influenced by meandering motions. Since submeso motions have time scales comparable with the averaging time (Anfossi et al. 2005; Mahrt 2007; Vickers and Mahrt 2007; Acevedo et al. 2014; Mortarini et al. 2016; Sect. 3.6) they can contribute both to the mean flow and to the horizontal velocity variances with significant sampling errors thus leading to the large scatter of the data. Concerning this issue, much longer averaging times (i.e., several hours) might not reduce the record-to-record variability (Mahrt et al. 2013).

\subsection{The Wind-Speed Effect on the Shear Stress}

Because the second-order moments are usually normalized with the shear stress, $\tau$, we consider the dependence on $U_{\mathrm{r}}$ of $2 E_{\mathrm{K}} / \tau$ and $\sigma_{w}^{2} / \tau$ (Fig. 6). In general, both depend on wind speed but with differences among the datasets.

For the Cabauw dataset (Fig. 6a), $2 E_{\mathrm{K}} / \tau$ increases sharply as $U_{\mathrm{r}} \rightarrow$ zero, indicating that the vertical transport of momentum decreases while TKE is maintained. A slight increase is observed also for the SABLES98 and UTP datasets (Fig. 6b, c), although there are few low-wind data for the first one. The high-wind values are consistent with those reported by Mauritsen and Svensson (2007) who, for near-neutral conditions, found $2 E_{\mathrm{K}} / \tau \approx 12$ : thus, although wind speed is not considered explicitly in their study, we argue that their observations correspond mainly to high-wind conditions.

For the same three datasets, $\sigma_{w}^{2} / \tau$ decreases as $U_{\mathrm{r}} \rightarrow$ zero, confirming that most of the TKE lies in the horizontal components for low-wind conditions, in agreement with the results shown by Fig. 4 . When recognizable, the increase in $2 E_{\mathrm{K}} / \tau$ and the decrease in $\sigma_{w}^{2} / \tau$ occur at the same $U_{\mathrm{r}}$ found for $P$ and $A_{w}$.

The picture is partly different for the CCT dataset (Fig. 6d). On average, $2 E_{\mathrm{K}} / \tau$ is nearly constant and $\sigma_{w}^{2} / \tau$ gently decreases with decreasing wind speed, both showing large recordto-record variability. We cannot exclude that the low-wind behaviour of $\sigma_{w}^{2} / \tau$ is influenced by instrumental errors, because both $\sigma_{w}^{2}$ and $\tau$ contain vertical velocity fluctuations that (contrary to horizontal ones) can become very small, as indicated by Fig. 4. 
(a) Cabauw $z=3 \mathrm{~m}$

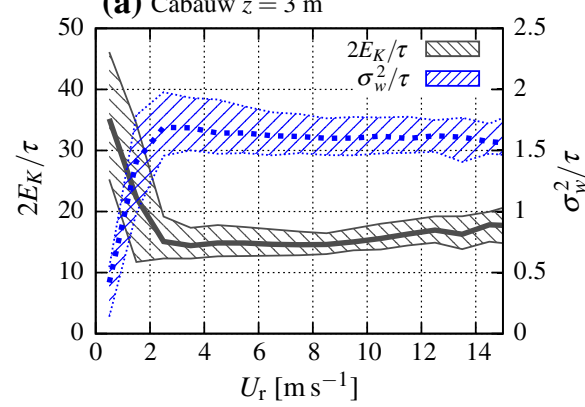

(c) $\mathrm{UTP} z=9 \mathrm{~m}$

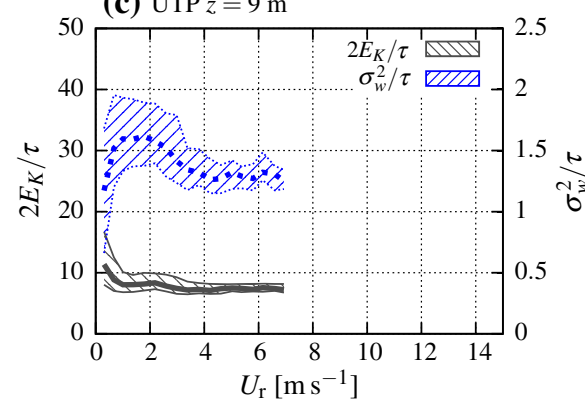

(b) SABLES $98 z=5.8 \mathrm{~m}$

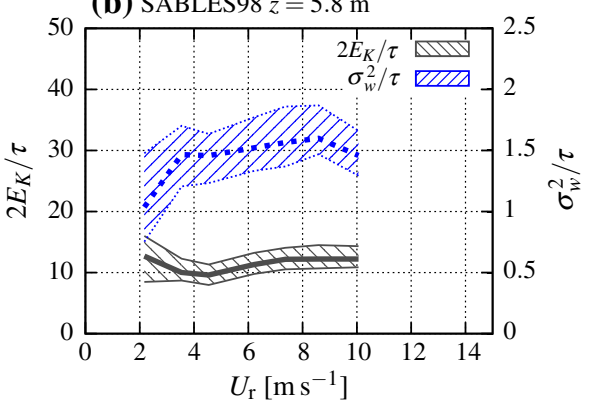

(d) $\mathrm{CCT} z=7.5 \mathrm{~m}$

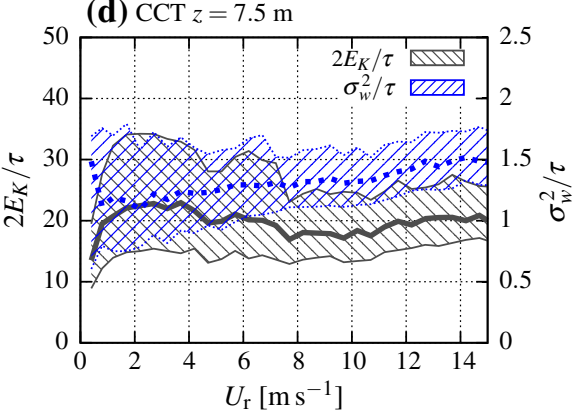

Fig. $62 E_{\mathrm{K}} / \tau$ (grey, solid) and $\sigma_{w}^{2} / \tau$ (blue, dashed) versus $U_{\mathrm{r}}$ : median and 25th-75th percentile range (for the other measurement levels see Online Resource 5)

\subsection{Hints from the Spectra}

To relate the observed behaviour to spectral features and to parametrize this, we investigate the spectra of the three velocity components. Anfossi et al. (2005) showed that, in low-wind conditions, the spectrum of the cross-wind component has its maximum in the low-frequency range, giving rise to the so-called "meandering" (Mahrt 2007; Mortarini et al. 2013, 2016). Starting from the same evidence, Mortarini and Anfossi (2015) proposed a new empirical formulation for the spectra of the horizontal velocity components accounting for this lowfrequency contribution that, although expected also for neutral stratification (Bradshaw 1978), is not considered in classical formulations for the neutral surface layer (e.g., Kaimal et al. 1972). Although a detailed spectral analysis is beyond our scope, findings concerning the velocity spectra at the CCT site are presented below.

Power spectra for the three velocity components, $S_{\alpha}(n)$, are defined such that

$$
\int_{0}^{\infty} S_{\alpha}(n) d n=\sigma_{\alpha}^{2}
$$

for $\alpha=u, v, w$, where $n$ is the time frequency in $\mathrm{s}^{-1}$. After linear detrending of 10-Hz data, we calculated $S_{\alpha}(n)$ for near-neutral, 30-min records (as defined in Sect. 3.3) acquired at the CCT site at $z=7.5 \mathrm{~m}$. We chose this record length to represent properly the submeso contribution without encompassing significant changes of the ABL state. 
Table 3 Number of spectra belonging to each wind-speed class shown in Fig. 7 (each class is $2 \mathrm{~m} \mathrm{~s}^{-1}$ wide and centred around the reported value)

\begin{tabular}{lll}
\hline$\alpha$ & $C_{\alpha}$ & $D_{\alpha}$ \\
\hline$u$ & 102 & 33 \\
$v$ & 17 & 9.5 \\
$w$ & 4.3 & 4.2 \\
\hline
\end{tabular}

\begin{tabular}{ll}
\hline$U\left(\mathrm{~m} \mathrm{~s}^{-1}\right)$ & Number of spectra \\
\hline 2 & 117 \\
4 & 229 \\
8 & 375 \\
12 & 550 \\
\hline
\end{tabular}

Table 4 Coefficients of the spectral model, Eq. 7, represented in Fig. 7

Spectra were then normalized by $(\kappa \varepsilon z)^{2 / 3}$, estimating the TKE dissipation rate (for each 30-min record) as

$$
\varepsilon=\frac{2 \pi}{U} \overline{\left(\frac{n^{\frac{5}{3}} S_{u}(n)}{\alpha_{1}}\right)^{\frac{3}{2}}},
$$

where $\alpha_{1}=0.5$ (e.g., Olesen et al. 1984) and the overline indicates averaging over the frequency range $U / z<n<4 \mathrm{~s}^{-1}$. We chose the streamwise velocity spectrum because, having the longest sampled inertial subrange, it gives the most reliable estimate of $\varepsilon$ (Louis et al. 1983; Yadav et al. 1996). The lower boundary of the averaging interval corresponds to the non-dimensional frequency $f \equiv n z / U=1$, which is roughly the low-frequency end of the inertial subrange (e.g., Kaimal and Finnigan 1994), while the further limitation $n<4 \mathrm{~s}^{-1}$ avoids aliasing effects.

CCT velocity spectra normalized in the inertial subrange are shown in Fig. 7. To investigate the wind-speed effect, different wind-speed classes are considered (Table 3): median spectra are presented for each class, while record-to-record variability (i.e., 25th-75th percentile range) is shown only for the lowest and the highest wind speeds. As a reference, we consider the "blunt model" (e.g.,Olesen et al. 1984)

$$
\frac{n S_{\alpha}(n)}{(\kappa \varepsilon z)^{2 / 3}}=\frac{C_{\alpha} f}{\left(1+D_{\alpha} f\right)^{5 / 3}}
$$

where, accounting for the inertial-subrange behaviour,

$$
\begin{aligned}
C_{u} & =D_{u}^{5 / 3} \alpha_{1}(2 \pi \kappa)^{-2 / 3}, \\
C_{v, w} & =\frac{4}{3} D_{v, w}^{5 / 3} \alpha_{1}(2 \pi \kappa)^{-2 / 3} .
\end{aligned}
$$

The values of $C_{\alpha}$ and $D_{\alpha}$ are reported in Table 4; $C_{u, v}$ and $D_{u, v}$ are from the neutral Kansas spectra (Kaimal and Finnigan 1994). Because the "pointed" model is used for the vertical spectrum in the Kansas formulations instead of the "blunt" model considered here, $C_{w}$ and $D_{w}$ were estimated by fitting all the points shown in Fig. 7c with Eq. 7. Remarkably, the values obtained for $C_{w}$ and $D_{w}$ (Table 4 ) are close to those found by Olesen et al. (1984), their Eq. (19).

For the horizontal components, two frequency ranges can be recognized (Fig. 7a, b). A high-frequency range, for $f \gtrsim 10^{-1}$, where the spectra collapse on Kansas formulations, 
Fig. 7 Normalized power spectra of the streamwise (a), the cross-wind (b), and the vertical velocity component (c) for different wind-speed classes (Table 3): median values (points) are shown for each wind-speed class; 25 th-75th percentile range is shown for $2 \mathrm{~m} \mathrm{~s}^{-1}$ and $12 \mathrm{~m} \mathrm{~s}^{-1}$ only (shaded areas). Equation 7, with coefficients from Table 4, is also represented (solid line)

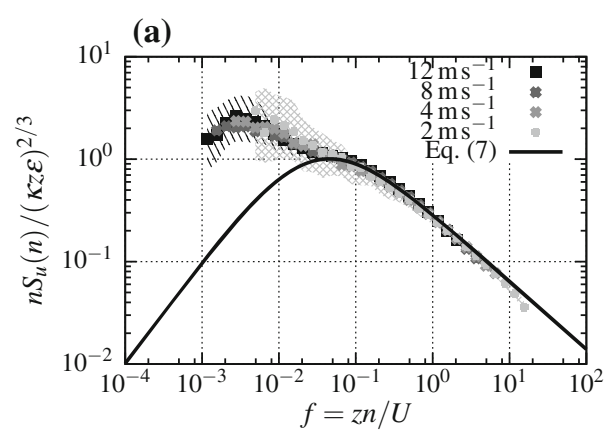

(b)

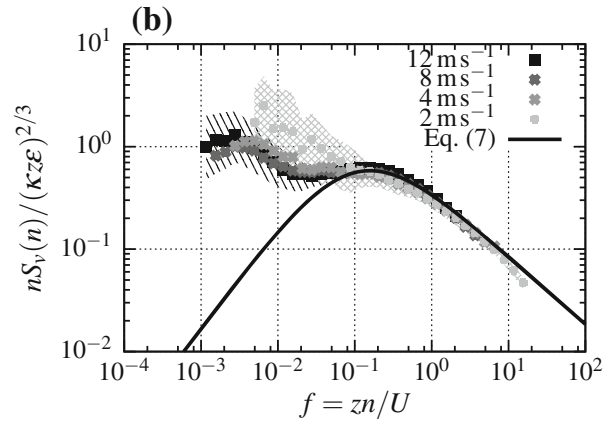

(c)

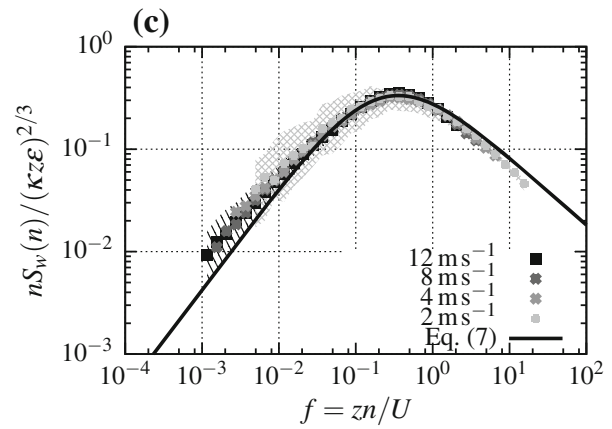

independently of wind speed. A low-frequency range, for $f \lesssim 10^{-1}$, where spectral levels are higher than those predicted by the neutral Kansas spectra. For the cross-wind component (Fig. 7b), the normalized spectrum increases substantially in the low-frequency range as $U$ decreases from 4 to $2 \mathrm{~ms}^{-1}$. This is consistent with a weak wind-speed dependence of lowfrequency spectral levels and a decrease in high-frequency spectral levels with decreasing wind speed, that was observed also by Anfossi et al. (2005).

Especially with low wind speeds, the 30-min record can be too short for a fair evaluation of the submeso contribution and of its eventual characteristic frequency, whose existence is suggested by the spectral peak observed for high wind speeds. Furthermore, the timespace transformation employing Taylor's hypothesis is unreliable for low wind speeds since turbulence intensity may be $>0.5$ (Sect. 3.2). However, despite these limitations, spectra indicate that the submeso contribution is relevant (or even dominant) in determining the horizontal shares, contrary to what is expected from classical surface-layer formulations. 
Table 5 Distribution of (near-neutral) low- and high-wind records among night, day, and morning/evening transitions, for the datasets of Cabauw, UTP, and CCT (for transition months only)

\begin{tabular}{llllcrr}
\hline Dataset & Wind & Night $(\%)$ & Day $(\%)$ & Morning $(\%)$ & Evening $(\%)$ & Total number \\
\hline Cabauw & Low & 36 & 33 & 17 & 14 & 72 \\
& High & 44 & 43 & 8 & 5 & 2075 \\
\multirow{3}{*}{ UTP } & Low & 64 & 12 & 17 & 7 & 211 \\
& High & 27 & 58 & 3 & 12 & 673 \\
CCT & Low & 43 & 39 & 10 & 7 & 122 \\
& High & 48 & 37 & 7 & 8 & 1812 \\
\hline
\end{tabular}

The picture is different for the vertical velocity component (Fig. 7c). Indeed, for any wind speed, vertical spectra are well described by the classical surface-layer formulations also in the low-frequency range, because large-scale vertical motions are inhibited by the presence of the ground (Olesen et al. 1984).

Summarizing, velocity spectra show a well-defined inertial subrange, independent of wind speed. However, a significant submeso contribution characterizes the $u$ and $v$ spectra, consistent with the behaviour shown by Figs. 4 and 5 and, in particular, with the large variability of the horizontal shares. Indeed, this contribution is poorly-represented in 10-min or 5-min statistics involving the horizontal velocity components (the requirement of Reynolds averaging being not fulfilled) and its relative amount (compared with that of small-scale turbulence) increases for diminishing wind speed (Fig. 7b).

\subsection{Time Distribution of Low-Wind, Near-Neutral Conditions}

We investigate here whether near-neutral, low-wind conditions occur mainly in particular times of the day or in particular seasons. At mid-latitudes (i.e., at the Cabauw, SABLES98 and UTP sites) the ABL is influenced by the daily cycle of sunlight while in the Arctic (i.e., at the CCT site) the seasonal cycle is dominant, the daily one playing a role during the transition months (i.e., March, April, September and October). Thus, we classified lowand high-wind records according to their acquisition time in "day", "night", "morning", and "evening" conditions. Table 5 shows this distribution for the datasets of the Cabauw, UTP, and CCT sites (considering transition months only), while the small number of cases hindered its evaluation for the SABLES98 dataset, which is thus not reported. Considering the time evolution of the fair-weather ABL (Stull 1988), the "morning" is supposed to last from the sunrise to $2 \mathrm{~h}$ later, while the "evening", from $1 \mathrm{~h}$ before to $1 \mathrm{~h}$ after sunset. No distinction is made between clear and cloudy conditions.

If low-wind (high-wind) 10-min records are uniformly distributed over the day, the expected frequencies would be $83 \%$ for day or night conditions and $17 \%$ for morning or evening transitions (because transitions account for $4 \mathrm{~h}$ in a day). These values are close to the observed ones for the CCT dataset (Table 5) indicating that the occurrence of both low- and high-wind conditions is independent of the time of the day at this site. However, at the Cabauw and UTP sites, low- and high-wind records are not uniformly distributed over the day. Specifically, morning and evening hours are the most characterized by low-wind conditions at the Cabauw site, while this is true for night and morning hours at the UTP site.

Accounting for the seasonal cycle, Fig. 8 shows the monthly distribution of low- and high-wind records for the CCT site indicating months belonging to polar night, polar day, 
(a) Low-wind

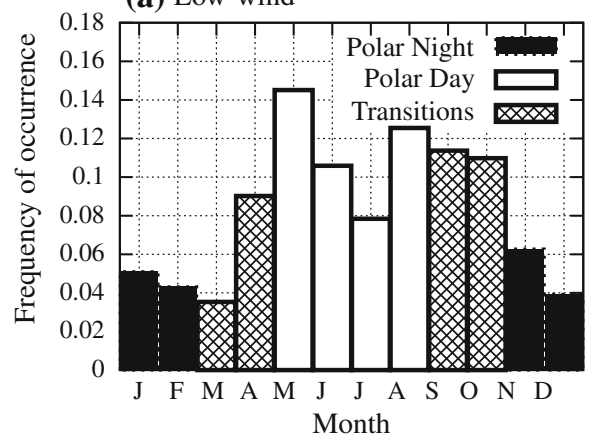

(b) High-wind

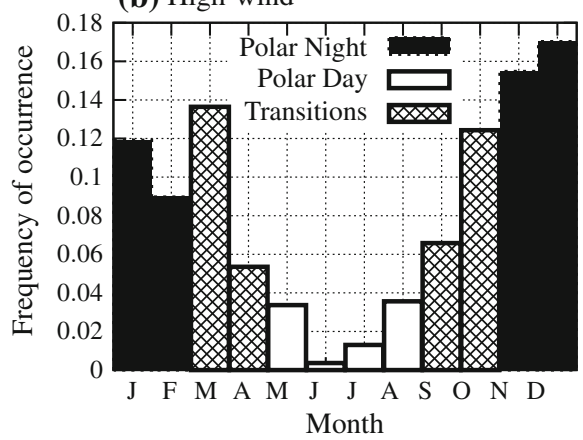

Fig. 8 Monthly distribution of a low-wind (555 10-min records), and b high-wind conditions (6652 10-min records) at the CCT site. Polar night (black), polar day (white) and transition months (dashed) are also indicated

and transition periods. Whereas low wind speeds correspond mostly to polar-day conditions (Fig. 8a), high wind speeds occur mainly during the polar night (Fig. 8b): the two distributions reflecting the seasonal variation of wind speed at Ny-Ålesund (Maturilli et al. 2013; Mazzola et al. 2016).

\section{Modelling Implications}

The presented results have impacts on the model parametrizations for the second-order moment equations. Let us consider the budget equations for the variance of the three velocity components $\sigma_{\alpha}^{2}(\alpha=u, v, w)$ assuming no directional shear of the velocity vector and neutral stratification (e.g., Zilitinkevich et al. 2013)

$$
\begin{array}{r}
Z_{u}=2 \tau \frac{\partial U}{\partial z}+Q_{u}-\frac{2}{3} \varepsilon, \\
Z_{v}=Q_{v}-\frac{2}{3} \varepsilon
\end{array}
$$

and

$$
Z_{w}=Q_{w}-\frac{2}{3} \varepsilon
$$

where $Q_{\alpha}(\alpha=u, v, w)$ are the pressure-redistribution terms (such that $Q_{u}+Q_{v}+Q_{w}=0$ ) and $Z_{\alpha}$ accounts for all the contributions not explicitly written in Eqs. 9-11: e.g., unsteadiness, advection and third-order terms.

For modelling, the TKE dissipation rate, $\varepsilon$, can be written as

$$
\varepsilon=\frac{u_{\mathrm{T}}^{3}}{l_{\mathrm{T}}}=\frac{u_{\mathrm{T}}^{2}}{t_{\mathrm{T}}},
$$

where $u_{\mathrm{T}}$ is a turbulent velocity, $l_{\mathrm{T}}$ is a length scale, and $t_{\mathrm{T}}=l_{\mathrm{T}} / u_{\mathrm{T}}$ is a derived time scale. A common choice is $u_{\mathrm{T}} \propto E_{\mathrm{K}}$ and $l_{\mathrm{T}} \propto z$ for near-neutral conditions (see e.g., Yamada and Mellor 1975; Nieuwstadt 1984; Nakanishi 2001; Zilitinkevich et al. 2013). However, the horizontal velocity variances (and thus the TKE) are affected by a significant (even dominant) submeso contribution (the relative amount may depend on wind speed), while the dissipation is related to the small scales. Thus, a more robust choice is $u_{T} \propto \sigma_{w}$, because submeso 
motions negligibly affect the vertical spectrum. Accordingly, the dissipation rate is written as

$$
\varepsilon=\frac{\sigma_{w}^{2}}{t_{\mathrm{T}}},
$$

where $t_{\mathrm{T}}=c_{w} z / \sigma_{w}$ with $c_{w} \approx 1$ (since $c_{w}=\sqrt{2} D_{w} \alpha_{1}^{3 / 2} / \pi=0.16 D_{w}$, as can be obtained integrating Eq. 7 for $\alpha=w$ ). A similar parametrization was adopted by Hunt et al. (1987) for shear-free conditions.

The TKE budget equation is obtained by summing Eqs. 9-11

$$
Z_{\mathrm{K}}=\tau \frac{\partial U}{\partial z}-\varepsilon
$$

where $Z_{\mathrm{K}} \equiv\left(Z_{u}+Z_{v}+Z_{w}\right) / 2$.

For the sake of completeness, the equation for the shear stress, $\tau$, reads

$$
Z_{\tau}=\sigma_{w}^{2} \frac{\partial U}{\partial z}-\varepsilon_{\tau}
$$

where $Z_{\tau}$ accounts for all neglected contributions and $\varepsilon_{\tau}$ is the effective dissipation rate (Zilitinkevich and Esau 2007) that can be expressed as (Zilitinkevich et al. 2013)

$$
\varepsilon_{\tau}=\frac{\tau}{c_{\tau} t_{\mathrm{T}}}
$$

Equation 15 is a starting point for future research (as discussed below), highlighting the coupling among the mean shear, the second-order moments, and the dissipation rate.

To obtain an expression for $A_{\alpha}$, a suitable parametrization for $Q_{\alpha}$ is needed. Rotta (1951) modelled $Q_{\alpha}$ using the return-to-isotropy assumption, from which $Q_{\alpha}$ is also known as the "return-to-isotropy" term. Starting from the Rotta (1951) expression, Zilitinkevich et al. (2013) proposed a new parametrization for neutral and stable stratification, accounting for the dependence of $A_{\alpha}$ on stability as observed in the atmospheric surface layer. The neutral limit for their expressions is

$$
\begin{gathered}
Q_{u}=-\frac{c_{\mathrm{R}}+1}{t_{\mathrm{T}}}\left[\sigma_{u}^{2}-\frac{2}{3} E_{\mathrm{K}}\right]-\frac{2}{3} \frac{c_{\mathrm{R}} c_{1}}{t_{\mathrm{T}}} E_{\mathrm{K}}, \\
Q_{v}=-\frac{c_{\mathrm{R}}+1}{t_{\mathrm{T}}}\left[\sigma_{v}^{2}-\frac{2}{3} E_{\mathrm{K}}\right]+\frac{2}{3} \frac{c_{\mathrm{R}} c_{1}}{t_{\mathrm{T}}} E_{\mathrm{K}}
\end{gathered}
$$

and

$$
Q_{w}=-\frac{c_{\mathrm{R}}+1}{t_{\mathrm{T}}}\left[\sigma_{w}^{2}-\frac{2}{3} E_{\mathrm{K}}\right] .
$$

The constants $c_{\mathrm{R}}$ and $c_{1}$ are independent of wind speed and Rotta's parametrization (Rotta 1951 ) is obtained taking $c_{1}=0$. Since the total (horizontal) variances enter in Eqs. 17-19, the same parametrization is used for the submeso motions and the small-scale eddies, and this can be questionable.

The expressions for the TKE shares are obtained from Eqs. 9-11, 13, and 17-19. Defining the non-dimensional terms

$$
\zeta_{\alpha} \equiv Z_{\alpha} \frac{t_{\mathrm{T}}}{\sigma_{w}^{2}}=Z_{\alpha} \frac{z}{\sigma_{w}^{3}}=\frac{Z_{\alpha}}{\varepsilon},
$$


for $\alpha=u, v, w$, we have

$$
\begin{array}{r}
A_{w}^{-1}=\frac{3 \zeta_{w}}{c_{\mathrm{R}}+1}+A_{w \infty}^{-1}, \\
A_{u}=\frac{1}{3}\left(1+A_{1}+A_{2}+A_{3}+A_{4}\right)
\end{array}
$$

and

$$
A_{v}=\frac{1}{3}\left(1-A_{1}-A_{2} / 2-A_{3}\right)
$$

where

$$
\begin{array}{r}
A_{1} \equiv-\frac{c_{R} c_{1}}{c_{R}+1}, \\
A_{2} \equiv \frac{4}{c_{R}+1} A_{w}, \\
A_{3} \equiv \frac{3}{c_{R}+1} \zeta_{v} A_{w}, \\
A_{4} \equiv \frac{3}{c_{R}+1} \zeta_{w} A_{w}
\end{array}
$$

and

$$
A_{w \infty}=\frac{1}{3}\left(\frac{c_{\mathrm{R}}+1}{c_{\mathrm{R}}+5 / 3}\right),
$$

the latter being the high-wind value of $A_{w}$, for which $\zeta_{w}=0$ is assumed. In the same limit, $\zeta_{v}=0$, having thus

$$
A_{u \infty}=\frac{1}{3}\left(1+A_{1}+\frac{4}{c_{\mathrm{R}}+1} A_{w \infty}\right)
$$

and

$$
A_{v \infty}=\frac{1}{3}\left(1-A_{1}-\frac{2}{c_{\mathrm{R}}+1} A_{w \infty}\right) .
$$

The value of $c_{\mathrm{R}}$ and $c_{1}$ can be obtained from Eqs. 28-30 with the observed high-wind values of $A_{\alpha}$ (Table 2): taking $A_{u \infty}=0.6, A_{v \infty}=0.3$, and $A_{w \infty}=0.1$, we find $c_{\mathrm{R}}=-0.7$ and $c_{1}=-0.24$.

Equations 21-23 relate the deviation of $A_{\alpha}$ from the high-wind value (Eqs. 28-30) to the magnitude of the $\zeta$-terms that can be expressed as a function of the wind speed, taking $\zeta_{\alpha}\left(U_{\mathrm{r}}\right)$. Because the scatter of $A_{w}$ is small (Fig. 4), empirical expressions for $\zeta_{w}\left(U_{\mathrm{r}}\right)$ can be derived from Eq. 28 and the observed variation of $A_{w}$ with $U_{\mathrm{r}}$. On the contrary, $\zeta_{v}$ (or $\zeta_{u}$ ) is unpredictable for each single realization because of the large scatter of $A_{u}$ and $A_{v}$ in low-wind conditions, but an expression can be obtained accounting for the average behaviour.

As an example, we consider the dependence of $A_{\alpha}$ on $U_{r}$ observed at the Cabauw and CCT sites. In Fig. 9, the median values already shown in Fig. 4a and d are reported together with the behaviour obtained from Eqs. 21-23 taking $\zeta_{\alpha}\left(U_{\mathrm{r}}\right)=\zeta_{\alpha 0} \exp \left(-a U_{\mathrm{r}}\right)$ for $\alpha=v, w$, where $\zeta_{\alpha 0}$ and $a$ are site-dependent. Without a rigorous derivation and only for capturing the broad behaviour, we chose $a=2 \mathrm{sm}^{-1}, \zeta_{v 0}=-8$, and $\zeta_{w 0}=8$ for the Cabauw site (Fig. 9a), and $a=1 \mathrm{~s} \mathrm{~m}^{-1}, \zeta_{v 0}=-0.5$, and $\zeta_{w}=0$ for the CCT site (Fig. 9b). Although qualitative, this example highlights that terms accounting for non-ideal (submeso) effects 
(a) Cabauw $z=3 \mathrm{~m}$

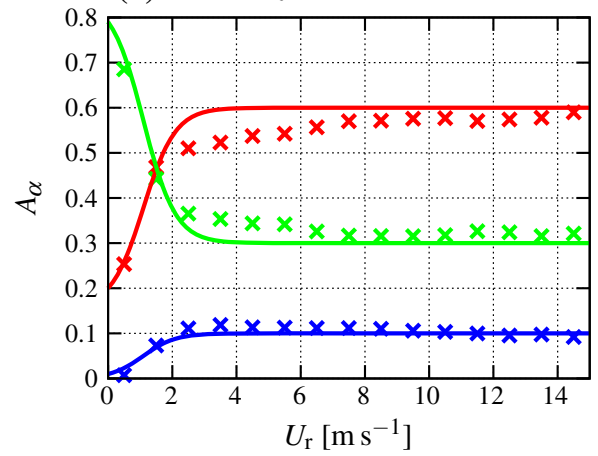

(b) $\mathrm{CCT} z=7.5 \mathrm{~m}$

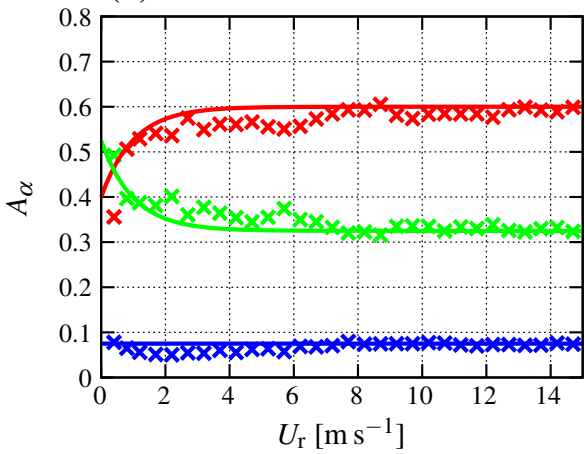

Fig. $9 A_{\alpha}$ versus $U_{\mathrm{r}}$ from the proposed model (lines) and observations (points, corresponding to the median values shown in Fig. 4a and d) for the Cabauw (a) and CCT (b) sites

should be considered in any parametrization of the boundary-layer turbulence that aims to cover the full range of wind speeds.

\section{Conclusions}

The share of the TKE among the three velocity components in the near-neutral surface layer was investigated for a wide range of wind speeds, using observations acquired over homogeneous and heterogeneous surfaces and with different environmental conditions. While our results are consistent with many previous studies, which found a significant effect of the wind speed on the velocity variances, the novelty of our approach is to single out the windspeed effect from the stability effect through the limitation of the analysis to near-neutral conditions. This further suggests that another parameter, beside atmospheric stability, should be taken into account in surface-layer parametrizations.

The main result is that the TKE shares are affected by the wind speed. Indeed, below a characteristic wind speed, which turns out to depend on the site: (i) the vertical share decreases, the TKE being more contained in the horizontal components; (ii) the turbulence becomes almost horizontally isotropic on average, but with large record-to-record variability for a given site; (iii) the TKE normalized over the shear stress increases. Features (i) and (iii) are consistent with an increasing contribution to surface-layer turbulence of almost twodimensional, submeso motions, with less effective vertical transfer of momentum, although we cannot establish, in general, if these motions are really "inactive" in the surface layer, in the meaning of Townsend (1961) and Bradshaw (1967). Being the vertical transfer of momentum commonly associated with the mean shear, feature (iii) is consistent with the observation that the friction velocity cannot be used as a turbulent velocity scale in low-wind conditions.

These effects are clearly found at the two horizontally homogeneous sites of Cabauw and SABLES98 while they are less clear at the heterogeneous CCT site (with complex topography), where the contribution from the effects related to non-ideal conditions seems to increase the spread of the data. Instead, the behaviour observed at the suburban UTP site is similar to that found at the more homogeneous sites, suggesting that the nature of the heterogeneity (isolated buildings, in this case) should be considered too. This confirms that, 
for a site with isolated obstacles such as that of UTP, the effect of the (low) wind speed dominates the flow dynamics with respect to the complex geometry (Trini Castelli et al. 2014).

Qualitatively, a similar tendency towards horizontal isotropy is found in convective conditions, supporting the view that the observed behaviour is related to the decreasing importance of shear-generated turbulence and the increased effect of submeso eddies. Furthermore, an analogy exists also with conditions of strong stable stratification, characterized by a large scatter of the shares and of the TKE over the shear stress, and a broad tendency towards horizontal isotropy: stability inhibits the vertical transport of momentum so that the horizontal shares can be increasingly affected by the submeso motions. All these observations lead to the conclusion that the dependence of the shares on the wind speed must also be accounted for, in the data analysis and for modelling, as it is done for the stability.

Spectral analysis confirms the significant submeso contribution to the spectra of the horizontal velocity components, beside the existence of a well-defined inertial subrange, independently of wind speed. For low wind speed, submeso modes enter as poorly sampled contributions in the statistics (i.e., variances and spectra) of the horizontal velocity components, while leaving unaffected the vertical one: thus explaining the large record-to-record variability of the horizontal shares. This raises in turn the question whether the averaging time should be extended in these conditions to better sample the submeso contribution.

For the above reasons, the applicability of TKE-based parametrizations for the pressureredistribution and the dissipation terms in the budget equations of the velocity variances (and the use of Reynolds averaged equations too) becomes questionable for low wind speeds. Accounting for our findings, we suggested a formulation for the TKE shares obtained from the budget equations of the velocity variances by expressing the dissipation rate through the vertical velocity variance and using simple expressions to describe the deviation from ideal conditions as wind speed decreases.

Acknowledgements Thanks are due to: the SABLES98 team, Prof. J.L. Casanova (Director of CIBA), and Dr. J. Peláez (for his technical support); the Korean Polar Research Institute (KOPRI), in particular Dr. T. Choi, that provided CSAT3 CCT sonic data. CY has been funded by the Spanish Government, under MINECO projects CGL2015-65627-C3-3-R (MINECO/FEDER) and CGL2016-81828-REDT.

\section{References}

Acevedo OC, Costa FD, Oliveira PE, Puhales FS, Degrazia GA, Roberti D (2014) The influence of submeso processes on stable boundary layer similarity relationships. J Atmos Sci 71(1):207-225

Acevedo OC, Mahrt L, Puhales FS, Costa FD, Medeiros LE, Degrazia GA (2016) Contrasting structures between the decoupled and coupled states of the stable boundary layer. Q J R Meteorol Soc 142(695):693702

Anfossi D, Oettl D, Degrazia G, Ferrero E, Goulart A (2005) An analysis of sonic anemometer observations in low wind speed conditions. Boundary-Layer Meteorol 114:179-203

Bradshaw P (1967) "Inactive" motion and pressure fluctuations in turbulent boundary layers. J Fluid Mech 30(2):241-258

Bradshaw P (1978) Comments on horizontal velocity spectra in an unstable surface layer. J Atmos Sci 35(9):1768-1769

Cheng Y, Brutsaert W (2005) Fluxprofile relationships for wind speed and temperature in the stable atmospheric boundary layer. Boundary-Layer Meteorol 114:519-538

Cuxart J, Yagüe C, Morales G, Terradellas E, Orbe J, Calvo J, Fernandez A, Soler MR, Infante C, Buenestado P, Espinalt A, Joergensen HE, Rees JM, Vila J, Redondo JM, Cantalapiedra IR, Conangla L (2000) Stable atmospheric boundary-layer experiment in Spain (SABLES 98): a report. Boundary-Layer Meteorol 96:337-370 
Falabino S, Trini Castelli S (2017) Estimating wind velocity standard deviation values in the inertial sublayer from observations in the roughness sublayer. Meteorol Atmos Phys 129(1):83-98

de Franceschi M, Zardi D, Tagliazucca M, Tampieri F (2009) Analysis of second order moments in surface layer turbulence in an alpine valley. Q J R Meteorol Soc 135:1750-1765

Grachev AA, Andreas EL, Fairall CW, Guest PS, Persson POG (2013) The critical richardson number and limits of applicability of local similarity theory in the stable boundary layer. Boundary-Layer Meteorol 147:51-82

Ha KJ, Hyun YK, Oh HM, Kim KE, Mahrt L (2007) Evaluation of boundary layer similarity theory for stable conditions in cases-99. Mon Weather Rev 135(10):3474-3483

Hanna SR (1982) Application in air pollution modelling. D. Reidel Publishing Company, Dordrecht

Hanna SR (1983) Lateral turbulence intensity and plume meandering during stable conditions. J Clim Appl Meteorol 22(8):1424-1430

Hanna SR (1990) Lateral dispersion in light-wind stable conditions. Il Nuovo Cimento C 13:889-894

Högström U, Hunt JCR, Smedman AS (2002) Theory and measurements for turbulence spectra and variances in the atmospheric neutral surface layer. Boundary-Layer Meteorol 103:101-124

Howell JF, Mahrt L (1997) Multiresolution flux decomposition. Boundary-Layer Meteorol 83:117137

Howell JF, Sun J (1999) Surface-layer fluxes in stable conditions. Boundary-Layer Meteorol 90:495520

Hunt J (1984) Turbulence structure in thermal convection and shear-free boundary layers. J Fluid Mech 138:161-184

Hunt J, Spalart P, Mansour N (1987) A general form for the dissipation length scale in turbulent shear flows. In: Studying turbulence using numerical simulation databases. Proceedings of the 1987 Summer Program, Stanford University, pp 179-184

Isichenko MB (1992) Percolation, statistical topography, and transport in random media. Rev Modern Phys 64(4):961-1043

Joffre SM, Laurila T (1988) Standard deviations of wind speed and direction from observations over a smooth surface. J Appl Meteorol 27(5):550-561

Kaimal J (1978) Horizontal velocity spectra in an unstable surface layer. J Atmos Sci 35(1):18-24

Kaimal JC, Finnigan JJ (1994) Atmospheric boundary layer flows. Their structure and measurement. Oxford University Press, Oxford

Kaimal JC, Wyngaard JC, Izumi Y, Coté OR (1972) Spectral characteristics of surface-layer turbulence. Q J R Meteorol Soc 98:563-589

Liang J, Zhang L, Wang Y, Cao X, Zhang Q, Wang H, Zhang B (2014) Turbulence regimes and the validity of similarity theory in the stable boundary layer over complex terrain of the Loess Plateau, China. J Geophys Res: Atmos 119:6009-6021. https://doi.org/10.1002/2014JD021510

Louis J, Weill A, Vidal-Madjar D (1983) Dissipation length in stable layers. Boundary-Layer Meteorol 25(3):229-243

Mahrt L (2007) Weak-wind mesoscale meandering in the nocturnal boundary layer. Envirion Fluid Mech 7(4):331-347

Mahrt L (2014) Stably stratified atmospheric boundary layers. Annu Rev Fluid Mech 46:23-45. https://doi. org/10.1146/annurev-fluid-010313-141354

Mahrt L, Richardson S, Seaman N, Stauffer DR (2012) Turbulence in the nocturnal boundary layer with light and variable winds. Q J R Meteorol Soc 138:1430-1439

Mahrt L, Thomas C, Richardson S, Seaman N, Stauffer DR, Zeeman M (2013) Non-stationary generation of weak turbulence for very stable and weak-wind conditions. Boundary-Layer Meteorol 147:179-199

Mahrt L, Sun J, Stauffer DR (2015) Dependence of turbulent velocities on wind speed and stratification. Boundary-Layer Meteorol 155:5571. https://doi.org/10.1007/s10546-014-9992-5

Maturilli M, Herber A, König-Langlo G (2013) Climatology and time series of surface meteorology in nyålesund, svalbard. Earth Syst Sci Data 5(1):155

Mauritsen T, Svensson G (2007) Observations of stably stratified shear-driven atmospheric turbulence at low and high Richardson numbers. J Atmos Sci 64:645-655

Mauritsen T, Svensson G, Zilitinkevich SS, Esau IN, Enger L, Grisogono B (2007) A total turbulent energy closure model for neutrally and stably stratified atmospheric boundary layers. J Atmos Sci 64:4113-4126

Mazzola M, Viola AP, Lanconelli C, Vitale V (2016) Atmospheric observations at the Amundsen-Nobile climate change tower in Ny-Ålesund Svalbard. Rendiconti Lincei 27(1):7-18

Monin A, Obukhov A (1954) Basic laws of turbulent mixing in the surface layer of the atmosphere. Contrib Geophys Inst Acad Sci USSR 151(163):e187

Monin AS, Yaglom AM (1971) Statistical fluid mechanics, vol I. MIT Press, Cambridge

Moraes OL, Acevedo OC, Degrazia GA, Anfossi D, da Silva R, Anabor V (2005) Surface layer turbulence parameters over a complex terrain. Atmos Environ 39:3103-3112 
Mortarini L, Anfossi D (2015) Proposal of an empirical velocity spectrum formula in low-wind speed conditions. Q J R Meteorol Soc 141(686):85-97

Mortarini L, Ferrero E, Falabino S, Trini Castelli S, Richiardone R, Anfossi D (2013) Low-frequency processes and turbulence structure in a perturbed boundary layer. Q J R Meteorol Soc 139(673):1059-1072

Mortarini L, Stefanello M, Degrazia G, Roberti D, Castelli ST, Anfossi D (2016) Characterization of wind meandering in low-wind-speed conditions. Boundary-Layer Meteorol 161(1):165-182

Nakanishi M (2001) Improvement of the mellor-yamada turbulence closure model based on large-eddy simulation data. Boundary-Layer Meteorol 99(3):349-378

Nieuwstadt FTM (1984) The turbulent structure of the stable, nocturnal boundary layer. J Atmos Sci 41:22022216

Obukhov A (1971) Turbulence in an atmosphere with a non-uniform temperature. Boundary-Layer Meteorol 2(1):7-29

Olesen HR, Larsen SE, Hojstrup J (1984) Modelling velocity spectra in the lower part of the planetary boundary layer. Boundary-Layer Meteorol 29:285-312

Pahlow M, Parlange M, Porte-Agel F (2001) On Monin-Obukhov similarity in the stable atmospheric boundary layer. Boundary-Layer Meteorol 99:225-248

Rotta JC (1951) Statisthe Theorie nichthomogener Turbulenz. Z Phys 129:547-572

San José R, Casanova J, Viloria RE, Casanova J (1985) Evaluation of the turbulent parameters of the unstable surface boundary layer outside Businger's range. Atmos Environ (1967) 19(10):1555-1561

Smith F, Abbott P (1961) Statistics of lateral gustiness at $16 \mathrm{~m}$ above ground. Q J R Meteorol Soc 87(374):549_ 561

Stull RB (1988) An introduction to boundary layer meteorology. Kluwer Academic Publishers, Alphen aan den Rijn

Sun J, Mahrt L, Banta RM, Pichugina YL (2012) Turbulence regimes and turbulence intermittency in the stable boundary layer during cases-99. J Atmos Sci 69(1):338-351

Sun J, Lenschow DH, LeMone MA, Mahrt L (2016) The role of large-coherent-eddy transport in the atmospheric surface layer based on cases-99 observations. Boundary-Layer Meteorol 160(1):83-111

Tampieri F (2017) Turbulence and dispersion in the planetary boundary layer. Springer International, Berlin

Taylor GI (1921) Diffusion by continuous movements. Proc Lond Math Soc 20:196-211

Thomson DJ (1987) Criteria for the selection of stochastic models of particle trajectories in turbulent flows. J Fluid Mech 180:529-556

Townsend A (1961) Equilibrium layers and wall turbulence. J Fluid Mech 11(1):97-120

Trini Castelli S, Falabino S (2013) Analysis of the parameterization for the wind-velocity fluctuation standard deviations in the surface layer in low-wind conditions. Meteorol Atmos Phys 119:91-107. https://doi. org/10.1007/s00703-012-0219-3

Trini Castelli S, Falabino S, Mortarini L, Ferrero E, Richiardone R, Anfossi D (2014) Experimental investigation of surface-layer parameters in low wind-speed conditions in a suburban area. Q J R Meteorol Soc 140(683):2023-2036

Troen I, Mahrt L (1986) A simple model of the atmospheric boundary layer: sensitivity to surface evaporation. Boundary-Layer Meteorol 37:129-148

Van Ulden A, Wieringa J (1996) Atmospheric boundary layer research at cabauw. In: Boundary-layer meteorology 25th anniversary vol, 1970-1995, Springer, pp 39-69

Verkaik J, Holtslag A (2007) Wind profiles, momentum fluxes and roughness lengths at cabauw revisited. Boundary-Layer Meteorol 122(3):701-719

Vickers D, Mahrt L (2003) The cospectral gap and turbulent flux calculations. J Atmos Ocean Technol 20:660672

Vickers D, Mahrt L (2006) A solution for flux contamination by mesoscale motions with very weak turbulence. Boundary-Layer Meteorol 118(3):431-447

Vickers D, Mahrt L (2007) observations of the cross-wind velocity variance in the stable boundary layer. Envirion Fluid Mech 7:55-71

Van de Wiel B, Moene A, Jonker H, Baas P, Basu S, Donda J, Sun J, Holtslag A (2012) The minimum wind speed for sustainable turbulence in the nocturnal boundary layer. J Atmos Sci 69(11):3116-3127

Wilson JD (2008) Monin-obukhov functions for standard deviations of velocity. Boundary-Layer Meteorol 129:353-369

Yadav AK, Raman S, Sharan M (1996) Surface layer turbulence spectra and dissipation rates during low winds in tropics. Boundary-Layer Meteorol 79(3):205-223

Yagüe C, Viana S, Maqueda G, Redondo JM (2006) Influence of stability on the flux-profile relationships for wind speed, $\phi_{m}$, and temperature, $\phi_{h}$, for the stable atmospheric boundary layer. Nonlinear Proc Geophys 13:185-203 
Yamada T, Mellor G (1975) A simulation of the Wangara atmospheric boundary layer data. J Atmos Sci 32(12):2309-2329

Zilitinkevich SS, Esau IN (2007) Similarity theory and calculation of turbulent fluxes at the surface for the stably stratified atmospheric boundary layer. Boundary-Layer Meteorol 125:193-205

Zilitinkevich SS, Hunt JCR, Esau IN, Grachev AA, Lalas DP, Akylas E, Tombrou M, Fairall CW, Fernando HJS, Baklanov A, Joffre SM (2006) The influence of large convective eddies on the surface-layer turbulence. Q J R Meteorol Soc 132:1423-1456

Zilitinkevich SS, Elperin T, Kleeorin N, Rogachevskii I, Esau IN (2013) A hierarchy of energy-and flux-budget (EFB) turbulence closure models for stably-stratified geophysical flows. Boundary-Layer Meteorol $146: 341-373$

Publisher's Note Springer Nature remains neutral with regard to jurisdictional claims in published maps and institutional affiliations. 